\title{
ENERGY AND VORTICITY OF THE GINZBURG-LANDAU MODEL WITH VARIABLE MAGNETIC FIELD
}

\author{
K. ATTAR
}

\begin{abstract}
We consider the Ginzburg-Landau functional with a variable applied magnetic field in a bounded and smooth two dimensional domain. The applied magnetic field varies smoothly and is allowed to vanish non-degenerately along a curve. Assuming that the strength of the applied magnetic field varies between two characteristic scales, and the Ginzburg-Landau parameter tends to $+\infty$, we determine an accurate asymptotic formula for the minimizing energy and show that the energy minimizers have vortices. The new aspect in the presence of a variable magnetic field is that the density of vortices in the sample is not uniform.
\end{abstract}

\section{INTRODUCTION}

We consider a bounded, open and simply connected set $\Omega \subset \mathbb{R}^{2}$ with smooth boundary. We suppose that $\Omega$ models a superconducting sample subject to an applied external magnetic field. The energy of the sample is given by the Ginzburg-Landau functional,

$$
\mathcal{E}_{\kappa, H}(\psi, \mathbf{A})=\int_{\Omega}\left(|(\nabla-i \kappa H \mathbf{A}) \psi|^{2}+\frac{\kappa^{2}}{2}\left(1-|\psi|^{2}\right)^{2}\right) d x+\kappa^{2} H^{2} \int_{\Omega}\left|\operatorname{curl} \mathbf{A}-B_{0}\right|^{2} d x .
$$

Here $\kappa$ and $H$ are two positive parameters, to simplify we will consider that $H=H(\kappa)$. The wave function (order parameter) $\psi \in H^{1}(\Omega ; \mathbb{C})$ and the magnetic potential $\mathbf{A} \in H_{\mathrm{div}}^{1}(\Omega)$. The space $H_{\text {div }}^{1}(\Omega)$ is defined in $(1.4)$ below. Finally, the function $B_{0} \in C^{\infty}(\bar{\Omega})$ gives the intensity of the external variable magnetic field. Let $\Gamma=\left\{x \in \bar{\Omega}, B_{0}(x)=0\right\}$, then, we assume that $B_{0}$ satisfies :

$$
\begin{cases}\left|B_{0}\right|+\left|\nabla B_{0}\right|>0 & \text { in } \bar{\Omega} \\ \nabla B_{0} \cdot \vec{n} \neq 0 & \text { on } \Gamma \cap \partial \Omega .\end{cases}
$$

The assumption in 1.2 implies that for any open set $\omega$ relatively compact in $\Omega$ the set $\Gamma \cap \omega$ will be either empty, or consists of a union of smooth curves. Here, the definition of the functional (1.1) is taken as in [5]. In [9], the scaling for the intensity of the external magnetic field (denoted by $h$ ) is different. We choose the scaling from [5 for convenience when estimating the ground state energy of the functional.

Let $\mathbf{F}: \Omega \rightarrow \mathbb{R}^{2}$ be the unique vector field such that,

$$
\operatorname{div} \mathbf{F}=0 \text { and } \operatorname{curl} \mathbf{F}=B_{0} \text { in } \Omega, \nu \cdot \mathbf{F}=0 \text { on } \partial \Omega .
$$

The vector $\nu$ is the unit interior normal vector of $\partial \Omega$. We define the space,

$$
H_{\text {div }}^{1}(\Omega)=\left\{\mathbf{A}=\left(\mathbf{A}_{1}, \mathbf{A}_{2}\right) \in H^{1}(\Omega)^{2}: \operatorname{div} \mathbf{A}=0 \text { in } \Omega, \mathbf{A} \cdot \nu=0 \text { on } \partial \Omega\right\} .
$$

Critical points $(\psi, \mathbf{A}) \in H^{1}(\Omega ; \mathbb{C}) \times H_{\text {div }}^{1}(\Omega)$ of $\mathcal{E}_{\kappa, H}$ are weak solutions of the Ginzburg-Landau equations,

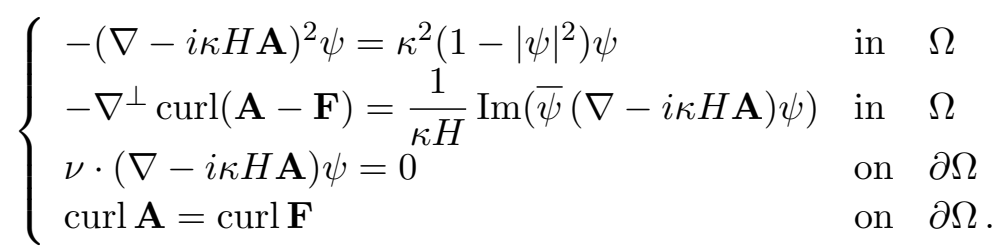


Here, $\operatorname{curl} \mathbf{A}=\partial_{x_{1}} \mathbf{A}_{2}-\partial_{x_{2}} \mathbf{A}_{1}$ and $\nabla^{\perp} \operatorname{curl} \mathbf{A}=\left(\partial_{x_{2}}(\operatorname{curl} \mathbf{A}),-\partial_{x_{1}}(\operatorname{curl} \mathbf{A})\right)$.

For a solution $(\psi, \mathbf{A})$ of 1.5 , the function $\psi$ describes the superconducting properties of the material and $(\kappa H$ curl $\mathbf{A})$ is the induced magnetic field. The number $\kappa$ is a parameter describing the properties of the material, and the number $H$ measures the variation of the intensity of the applied magnetic field. We focus on the regime of large values of $\kappa, \kappa \rightarrow+\infty$.

In this paper, we study the ground state energy defined as follows:

$$
\mathrm{E}_{\mathrm{g}}(\kappa, H)=\inf \left\{\mathcal{E}_{\kappa, H}(\psi, \mathbf{A}):(\psi, \mathbf{A}) \in H^{1}(\Omega ; \mathbb{C}) \times H_{\text {div }}^{1}(\Omega)\right\} .
$$

More precisely, we give an asymptotic estimate valid when $H(\kappa)$ satisfies:

$$
C_{\min } \kappa^{\frac{1}{3}} \leq H(\kappa) \ll \kappa \quad \text { as } \kappa \longrightarrow+\infty,
$$

where $C_{\min }$ is a positive constant.

The behavior of $\mathrm{E}_{\mathrm{g}}(\kappa, H)$ involves a function $\hat{f}:[0,1] \longrightarrow\left[0, \frac{1}{2}\right]$ introduced in 2.10 below. The function $\hat{f}$ is increasing, continuous and $\hat{f}(b)=\frac{1}{2}$, for all $b \geq 1$.

Under the assumption that $B_{0}(x)$ satisfies 1.2$)$ and that the function $H=H(\kappa)$ satisfies

$$
C_{1} \kappa \leq H \leq C_{2} \kappa,
$$

where $C_{1}$ and $C_{2}$ are positive constants, we obtained ${ }^{1}$ in [2] that

$$
\mathrm{E}_{\mathrm{g}}(\kappa, H)=\kappa^{2} \int_{\Omega} \hat{f}\left(\frac{H}{\kappa}\left|B_{0}(x)\right|\right) d x+o(\kappa H), \quad \text { as } \kappa \longrightarrow+\infty .
$$

In this paper, we generalize this result to the case when $H(\kappa)$ satisfies (1.7).

Theorem 1.1. Under Assumptions (1.2) and (1.7), the ground state energy in (1.6) satisfies, as $\kappa \longrightarrow+\infty$

$$
\mathrm{E}_{\mathrm{g}}(\kappa, H)=\kappa^{2} \int_{\Omega} \hat{f}\left(\frac{H}{\kappa}\left|B_{0}(x)\right|\right) d x+o\left(\kappa H \ln \frac{\kappa}{H}\right) .
$$

We will see in Remark 3.4 that the second term in the right hand side of (1.10), which is actually more simply $o(\kappa H \ln \kappa)$ when (1.7) is satisfied, is of lower order compared with the leading term. Actually (see in Theorem 2.1), the function $\hat{f}$ satisfies

$$
\hat{f}(b)=\frac{b}{2} \ln \frac{1}{b}(1+\hat{s}(b)), \quad \text { as } b \longrightarrow 0,
$$

with $\hat{s}(b)=o(1)$.

As a consequence of the behaviour of $\hat{f}$ above, (1.10) becomes

$$
\mathrm{E}_{\mathrm{g}}(\kappa, H)=\frac{1}{2} \kappa H\left[\int_{\Omega}\left|B_{0}(x)\right| \ln \frac{\kappa}{H\left|B_{0}(x)\right|} d x\right](1+o(1)) .
$$

When the magnetic field is constant (i.e $B_{0}$ is a constant function), (1.11) is proved in [11] under the relaxed condition

$$
\frac{\ln \kappa}{\kappa} \ll H \ll \kappa .
$$

The reason why we do not obtain (1.11) under the relaxed condition 1.12 is probably technical. The method is to construct test configurations with a Dirichlet boundary condition. We can not construct periodic configurations as in [11] because the magnetic field $B_{0}$ is variable. The approach used in the proof of Theorem 1.1 is close to that in [8] which studies the same problem when $\Omega \subset \mathbb{R}^{3}$ and $B_{0}$ is constant.

\footnotetext{
${ }^{1}$ After a change of notation
} 
Remark 1.2. Notice that when $H(\kappa)$ satisfies 1.8 we have

$$
o(\kappa H)=o\left(\kappa H\left(\left|\ln \frac{H}{\kappa}\right|+1\right)\right) .
$$

If we assume that there exist positive constants $C_{\min }$ and $C_{1}$ and $H(\kappa)$ satisfies

$$
C_{\min } \kappa^{\frac{1}{3}} \leq H(\kappa) \leq C_{1} \kappa,
$$

then 1.9 and 1.10 can be rewritten in a unique statement:

$$
\mathrm{E}_{\mathrm{g}}(\kappa, H)=\kappa^{2} \int_{\Omega} \hat{f}\left(\frac{H}{\kappa}\left|B_{0}(x)\right|\right) d x+o\left(\kappa H\left(\left|\ln \frac{H}{\kappa}\right|+1\right)\right) .
$$

Remark 1.3. When the set $\Gamma=\left\{x \in \bar{\Omega}, B_{0}(x)=0\right\}$ consists of a finite number of smooth curves and the intensity of the magnetic field $H$ satisfies $\kappa \ll H \leq \mathcal{O}\left(\kappa^{2}\right)$, then the energy $\mathrm{E}_{\mathrm{g}}(\kappa, H)$ in (1.1) is estimated in [7].

Theorem 1.1 admits the following corollary which is useful in the proof of Theorem 1.5 below. The content of Corollary 1.4 gives us that the magnetic energy is small compared with the leading term in 1.14.

Corollary 1.4. Suppose that the assumptions of Theorem 1.1 hold. Then, the magnetic energy of the minimizer satisfies

$$
(\kappa H)^{2} \int_{\Omega}\left|\operatorname{curl} \mathbf{A}-B_{0}\right|^{2} d x=o\left(\kappa H \ln \frac{\kappa}{H}\right), \quad \text { as } \kappa \longrightarrow+\infty .
$$

If $(\psi, \mathbf{A}) \in H^{1}(\Omega ; \mathbb{C}) \times H_{\text {div }}^{1}(\Omega)$, we introduce the energy density,

$$
e(\psi, \mathbf{A})=|(\nabla-i \kappa H \mathbf{A}) \psi|^{2}+\frac{\kappa^{2}}{2}\left(1-|\psi|^{2}\right)^{2} .
$$

We also introduce the local energy of $(\psi, \mathbf{A})$ in a domain $\overline{\mathcal{D}} \subset \Omega$ :

$$
\mathcal{E}_{0}(\psi, \mathbf{A} ; \mathcal{D})=\int_{\mathcal{D}} e(\psi, \mathbf{A}) d x
$$

Furthermore, we define the Ginzburg-Landau energy of $(\psi, \mathbf{A})$ in a domain $\overline{\mathcal{D}} \subset \Omega$ as follows,

$$
\mathcal{E}(\psi, \mathbf{A} ; \mathcal{D})=\mathcal{E}_{0}(u, \mathbf{A} ; \mathcal{D})+(\kappa H)^{2} \int_{\Omega}|\operatorname{curl}(\mathbf{A}-\mathbf{F})|^{2} d x
$$

If $\mathcal{D}=\Omega$, we sometimes omit the dependence on the domain and write $\mathcal{E}_{0}(\psi, \mathbf{A})$ for $\mathcal{E}_{0}(\psi, \mathbf{A} ; \Omega)$.

The next theorem gives a local version of Theorem 1.1 .

Theorem 1.5. Under Assumption (1.2), if $(\psi, \mathbf{A})$ is a minimizer of (1.1) and $\mathcal{D}$ is regular set such that $\overline{\mathcal{D}} \subset \Omega$, then the following is true.

(1) If $H(\kappa)$ satisfies (1.7), then,

$$
\mathcal{E}(\psi, \mathbf{A}, \mathcal{D}) \geq \kappa^{2} \int_{\mathcal{D}} \hat{f}\left(\frac{H}{\kappa}\left|B_{0}(x)\right|\right) d x+o\left(\kappa H \ln \frac{\kappa}{H}\right), \quad \text { as } \kappa \longrightarrow+\infty .
$$

(2) If $H(\kappa)$ satisfies

$$
C_{\min }^{1} \kappa^{\frac{3}{5}} \leq H \ll \kappa \quad \text { as } \kappa \longrightarrow+\infty
$$

where $C_{\min }^{1}$ is a positive constant, then

$$
\mathcal{E}(\psi, \mathbf{A}, \mathcal{D}) \leq \kappa^{2} \int_{\mathcal{D}} \hat{f}\left(\frac{H}{\kappa}\left|B_{0}(x)\right|\right) d x+o\left(\kappa H \ln \frac{\kappa}{H}\right), \quad \text { as } \kappa \longrightarrow+\infty .
$$


As a consequence of the proof of Theorem 1.1, the methods used in [11] allow us to obtain information regarding the distribution of vortices in $\Omega$. When the magnetic field is constant (i.e $B_{0}$ is a constant), it is proved in [1] that $\psi$ has vortices whose density tends to be uniform. In Section 7 we will prove that, if $(\psi, \mathbf{A})$ is a minimizer of $(1.1)$ and $B_{0}(x)$ is a variable magnetic field, then, $\psi$ has vortices that are distributed everywhere in $\Omega$ but with a non uniform density.

The next theorem was proved by E. Sandier and S. Serfaty in [11] when the magnetic field is constant $\left(B_{0}(x)=1\right)$.

Theorem 1.6. Suppose that Assumption (1.2) holds and that $H(\kappa)$ satisfies (1.7). Let $(\psi, \mathbf{A})$ be a minimizer of (1.1). Then there exists $m=m(\kappa)$ disjoint disks $\left(D_{i}\left(a_{i}, r_{i}\right)\right)_{i=1}^{m}$ in $\Omega$ such that, as $\kappa \longrightarrow+\infty$,

(1) $\sum_{i=1}^{m} r_{i} \leq(\kappa H)^{\frac{1}{2}}\left(\ln \frac{\kappa}{H}\right)^{-\frac{7}{4}} \int_{\Omega} \frac{1}{\sqrt{\left|B_{0}(x)\right|}} d x(1+o(1))$.

(2) $|\psi| \geq \frac{1}{2}$ on $\cup_{i} \partial D_{i}$.

(3) If $d_{i}=\operatorname{deg}\left(\frac{\psi}{|\psi|}, \partial D_{i}\right)$ is the winding number of $\frac{\psi}{|\psi|}$ on $\partial D_{i}$, then as $\kappa \longrightarrow+\infty$

$$
\mu_{\kappa}=\frac{2 \pi}{\kappa H} \sum_{i=1}^{m} d_{i} \delta_{a_{i}} \longrightarrow B_{0}(x) d x \quad \text { and } \quad\left|\mu_{\kappa}\right|=\frac{2 \pi}{\kappa H} \sum_{i=1}^{m}\left|d_{i}\right| \delta_{a_{i}} \longrightarrow\left|B_{0}(x)\right| d x,
$$

in the weak sense of measures ${ }^{2}$, where $d x$ is the Lebesgue measure on $\mathbb{R}^{2}$ restricted to $\Omega$.

The measure $\mu$ describes the distribution of vortices see Fig.1, and it is called the vorticity measure, the function $o(1)$ is bounded independently of the choice of the minimizer $(\psi, \mathbf{A})$.

Notation. Throughout the paper, we use the following notation:

- If $a(\kappa)$ and $b(\kappa)$ are two positive functions, we write $a(\kappa) \ll b(\kappa)$ if $a(\kappa) / b(\kappa) \rightarrow 0$ as $\kappa \rightarrow \infty$.

- If $a(\kappa)$ and $b(\kappa)$ are two functions with $b(\kappa) \neq 0$, we write $a(\kappa) \sim b(\kappa)$ if $a(\kappa) / b(\kappa) \rightarrow 1$ as $\kappa \rightarrow \infty$.

- If $a(\kappa)$ and $b(\kappa)$ are two positive functions, we write $a(\kappa) \approx b(\kappa)$ if there exist positive constants $c_{1}, c_{2}$ and $\kappa_{0}$ such that $c_{1} b(\kappa) \leq a(\kappa) \leq c_{2} b(\kappa)$ for all $\kappa \geq \kappa_{0}$.

- Given $R>0$ and $x=\left(x_{1}, x_{2}\right) \in \mathbb{R}^{2}, Q_{R}(x)=\left(-R / 2+x_{1}, R / 2+x_{1}\right) \times\left(-R / 2+x_{2}, R / 2+\right.$ $x_{2}$ ) denotes the square of side length $R$ centered at $x$ and we write $Q_{R}=Q_{R}(0)$.

\section{A REFERENCE PROBlem}

Consider two constants $b \in(0,1)$ and $R>0$. If $u \in H^{1}\left(Q_{R}\right)$, we define the following Ginzburg-Landau energy,

$$
F_{b, Q_{R}}^{\sigma}(u)=\int_{Q_{R}}\left(b\left|\left(\nabla-i \sigma \mathbf{A}_{0}\right) u\right|^{2}+\frac{1}{2}\left(1-|u|^{2}\right)^{2}\right) d x,
$$

where $\sigma \in\{-1,+1\}$ and

$$
\mathbf{A}_{0}(x)=\frac{1}{2}\left(-x_{2}, x_{1}\right), \quad \forall x=\left(x_{1}, x_{2}\right) \in \mathbb{R}^{2} .
$$

Notice that the magnetic potential $\mathbf{A}_{0}$ satisfies:

$$
\operatorname{curl} \mathbf{A}_{0}=1 \text { in } \mathbb{R}^{2} \text {. }
$$

\footnotetext{
${ }^{2} \mu_{\kappa}$ converge weakly to $\mu$ means that:

$$
\mu_{\kappa}(f) \longrightarrow \mu(f), \quad \forall f \in C_{0}(\Omega)
$$
}


We introduce the two ground state energies

$$
\begin{gathered}
e_{N}(b, R)=\inf \left\{F_{b, Q_{R}}^{+1}(u): u \in H^{1}\left(Q_{R} ; \mathbb{C}\right)\right\} \\
e_{D}(b, R)=\inf \left\{F_{b, Q_{R}}^{+1}(u): u \in H_{0}^{1}\left(Q_{R} ; \mathbb{C}\right)\right\} .
\end{gathered}
$$

The minimization of the functional $F_{b, Q_{R}}^{+1}$ over 'magnetic periodic' functions appears naturally in the proof. Let us introduce the following space

$$
E_{R}=\left\{u \in H_{\mathrm{loc}}^{1}\left(\mathbb{R}^{2} ; \mathbb{C}\right): u\left(x_{1}+R, x_{2}\right)=e^{i R \frac{x_{2}}{2}} u\left(x_{1}, x_{2}\right), u\left(x_{1}, x_{2}+R\right)=e^{-i R \frac{x_{1}}{2}} u\left(x_{1}, x_{2}\right)\right\}
$$

together with the ground state energy

$$
e_{p}(b, R)=\inf \left\{F_{b, Q_{R}}^{+1}(u): u \in E_{R}\right\} .
$$

Since $F_{b, Q_{R}}^{+1}$ is bounded from below, there exists for each $e_{\#}(b, R)$ with $\# \in\{N, D, p\}$, a ground state (minimizer). Note also that by comparison of the three domains of minimization it is clear that

$$
e_{N}(b, R) \leq e_{p}(b, R) \leq e_{D}(b, R)
$$

In the three cases, if $u$ is such a ground state, $u$ satisfies the Ginzburg-Landau equation

$$
b\left(\nabla-i \mathbf{A}_{0}\right)^{2} u=\left(1-|u|^{2}\right) u,
$$

and it results from a standard application of the maximum principle that

$$
|u| \leq 1 \text {. }
$$

As $F_{b, Q_{R}}^{+1}(u)=F_{b, Q_{R}}^{-1}(\bar{u})$, it is also immediate that,

$$
\inf _{u \in H^{1}\left(Q_{R} ; \mathbb{C}\right)} F_{b, Q_{R}}^{+1}(u)=\inf _{u \in H^{1}\left(Q_{R} ; \mathbb{C}\right)} F_{b, Q_{R}}^{-1}(u) .
$$

In the next theorem we will define the limiting function $\hat{f}$, which describes the ground state energy of both two and three dimensional superconductors subject to high magnetic fields (see [6]).

Theorem 2.1. Let $e_{p}(b, R)$ be as introduced in 2.6.

(1) For any $b \in[0, \infty)$, there exists a constant $\hat{f}(b) \geq 0$ such that

$$
\hat{f}(b)=\lim _{R \longrightarrow \infty} \frac{e_{p}(b, R)}{\left|Q_{R}\right|}=\lim _{R \longrightarrow \infty} \frac{e_{D}(b, R)}{\left|Q_{R}\right|} .
$$

(2) For all $b \geq 1, \hat{f}(b)=\frac{1}{2}$.

(3) The function $[0, \infty) \ni b \longmapsto \hat{f}(b)$ is continuous, non-decreasing and its range is the interval $[0,1 / 2]$.

(4) As $b \longrightarrow 0_{+}, \hat{f}(b)$ satisfies

$$
\hat{f}(b)=\frac{b}{2} \ln \frac{1}{b}(1+\hat{s}(b)),
$$

where the function $\hat{s}:(0,+\infty) \longmapsto(-\infty,+\infty)$ satisfies

$$
\lim _{b \longrightarrow 0} \hat{s}(b) \longrightarrow 0 \text {. }
$$

(5) There exist universal constants $C$ and $R_{0}$ such that

$$
\forall R \geq R_{0}, \quad \forall b \in[0,1], \quad\left|\hat{f}(b)-\frac{e_{p}(b, R)}{R^{2}}\right| \leq \frac{C}{R} .
$$


(6) There exist positive constants $b_{0}, R_{0}$ and a function

$$
\text { err }:(0,1) \times(0,+\infty) \longrightarrow(0,+\infty)
$$

such that

$$
\forall \epsilon \geq 0, \exists \eta \geq 0 \text { if }|b|+\frac{1}{R}<\eta \text { then }|\operatorname{err}(b, R)| \leq \epsilon
$$

and

$$
\forall b \in\left(0, b_{0}\right), \quad \forall R \in\left(R_{0},+\infty\right), \quad \frac{e_{N}(b, R)}{R^{2}} \geq \hat{f}(b)(1-\operatorname{err}(b, R)) .
$$

The limiting function $\hat{f}$ was defined in $([1],[10,[8])$. The estimate in $(2.11)$ and $(2.12)$ are obtained by Fournais-Kachmar (see [6, Theorem 2.1 and Proposition 2.8]) and by Kachmar (see [8, Theorem 2.4]) respectively. The lower bound in (2.15) is a consequence of [8, Theorem 2.1 and (2.9)].

We need the next proposition in the proof of the lower bound of $\mathcal{E}_{\kappa, H}(\psi, \mathbf{A})$.

Proposition 2.2. There exists a positive constant $C$, such that if

$$
R \geq 1 \quad \text { and } \quad 0<b<1,
$$

then,

$$
e_{D}(b, R) \leq e_{N}(b, R)+C R b^{\frac{1}{2}} .
$$

Proof. Without loss of generality, we can suppose $\sigma=+1$. Let $u \in H^{1}\left(Q_{R}\right)$ be a minimizer of the functional in (2.1), i.e. such that:

$$
e_{N}(b, R)=F_{b, Q_{R}}^{+1}(u)=\int_{Q_{R}}\left(b\left|\left(\nabla-i \mathbf{A}_{0}\right) u\right|^{2}+\frac{1}{2}\left(1-|u|^{2}\right)^{2}\right) d x .
$$

We introduce a cut-off function $\chi_{R, b} \in C_{c}^{\infty}\left(\mathbb{R}^{2}\right)$ such that

$$
0 \leq \chi_{R, b} \leq 1 \quad \text { in } \mathbb{R}^{2}, \quad \operatorname{supp} \chi_{R, b} \subset Q_{R}, \quad \chi_{R, b}=1 \quad \text { in } Q_{R-b^{\frac{1}{2}}} .
$$

In addition, the function $\chi_{R, b}$ can be chosen such that for some universal constants $C$ and $C^{\prime}$, we have,

$$
\left|\nabla \chi_{R, b}\right| \leq C b^{-\frac{1}{2}} \quad \text { and } \quad\left|\Delta \chi_{R, b}\right| \leq C^{\prime} b^{-1}, \quad \forall R \geq 1 \quad \text { and } \quad \forall b \in(0,1) .
$$

Let $u_{R, b}(x)=\chi_{R, b}(x) u(x)$. Then $u_{R, b} \in H_{0}^{1}\left(Q_{R}\right)$ and consequently

$$
e_{D}(b, R) \leq F_{b, Q_{R}}^{+1}\left(u_{R, b}\right) .
$$

We rewrite $F_{b, Q_{R}}^{+1}\left(u_{R, b}\right)$ as follows,

$$
\begin{aligned}
F_{b, Q_{R}}^{+1}\left(u_{R, b}\right) & =\int_{Q_{R}}\left(b\left|\left(\nabla-i \mathbf{A}_{0}\right) \chi_{R, b} u\right|^{2}+\frac{1}{2}\left(1-\left|\chi_{R, b} u\right|^{2}\right)^{2}\right) d x \\
& =\int_{Q_{R}}\left(b\left|\left(\nabla-i \mathbf{A}_{0}\right) \chi_{R, b} u\right|^{2}+\frac{1}{2}\left(1-2|u|^{2}+\left|\chi_{R, b} u\right|^{4}+2\left(|u|^{2}-\left|\chi_{R, b} u\right|^{2}\right)\right)\right) d x \\
& \leq \int_{Q_{R}}\left(b\left|\left(\nabla-i \mathbf{A}_{0}\right) \chi_{R, b} u\right|^{2}+\frac{1}{2}\left(1-|u|^{2}\right)^{2}\right) d x+\int_{Q_{R} \backslash Q_{R-b^{\frac{1}{2}}}}\left(1-\left|\chi_{R, b}\right|^{2}\right)|u|^{2} d x .
\end{aligned}
$$


We estimate from above the term $\int_{Q_{R}}\left|\left(\nabla-i \mathbf{A}_{0}\right) \chi_{R, b} u\right|^{2} d x$ as follows:

$$
\begin{aligned}
\int_{Q_{R}}\left|\left(\nabla-i \mathbf{A}_{0}\right) \chi_{R, b} u\right|^{2} d x= & \left\langle\left(\nabla-i \mathbf{A}_{0}\right) \chi_{R, b} u,\left(\nabla-i \mathbf{A}_{0}\right) \chi_{R, b} u\right\rangle \\
= & \left\langle\nabla \chi_{R, b} u+\chi_{R, b}\left(\nabla-i \mathbf{A}_{0}\right) u, \nabla \chi_{R, b} u+\chi_{R, b}\left(\nabla-i \mathbf{A}_{0}\right) u\right\rangle \\
= & \left\langle\nabla \chi_{R, b} u, \nabla \chi_{R, b} u\right\rangle+\left\langle\chi_{R, b}\left(\nabla-i \mathbf{A}_{0}\right) u, \chi_{R, b}\left(\nabla-i \mathbf{A}_{0}\right) u\right\rangle \\
& \quad+\left\langle\nabla \chi_{R, b} u, \chi_{R, b}\left(\nabla-i \mathbf{A}_{0}\right) u\right\rangle+\left\langle\chi_{R, b}\left(\nabla-i \mathbf{A}_{0}\right) u, \nabla \chi_{R, b} u\right\rangle .
\end{aligned}
$$

An integration by parts yields,

$$
\begin{aligned}
\left\langle\nabla \chi_{R, b} u, \chi_{R, b}\left(\nabla-i \mathbf{A}_{0}\right) u\right\rangle=- & \left\langle\nabla \chi_{R, b} u, \nabla \chi_{R, b} u\right\rangle \\
& -\left\langle\chi_{R, b} \Delta \chi_{R, b} u, u\right\rangle-\left\langle\chi_{R, b}\left(\nabla-i \mathbf{A}_{0}\right) u, \nabla \chi_{R, b} u\right\rangle,
\end{aligned}
$$

which implies that

$$
\int_{Q_{R}}\left|\left(\nabla-i \mathbf{A}_{0}\right) \chi_{R, b} u\right|^{2} d x=\left\langle\chi_{R, b}\left(\nabla-i \mathbf{A}_{0}\right) u, \chi_{R, b}\left(\nabla-i \mathbf{A}_{0}\right) u\right\rangle-\left\langle\chi_{R, b} \Delta \chi_{R, b} u, u\right\rangle .
$$

Putting 2.24 into 2.22, we get

$$
\begin{aligned}
F_{b, Q_{R}}^{+1}\left(u_{R, b}\right) \leq \int_{Q_{R}}\left(b\left|\chi_{R, b}\left(\nabla-i \mathbf{A}_{0}\right) u\right|^{2}\right. & \left.+\frac{1}{2}\left(1-|u|^{2}\right)^{2}\right) d x \\
& +\int_{Q_{R} \backslash Q_{R-b^{\frac{1}{2}}}}\left(1-\left|\chi_{R, b}\right|^{2}\right)|u|^{2} d x+b \int_{Q_{R} \backslash Q_{R-b^{\frac{1}{2}}}}\left|\Delta \chi_{R, b}\right||u|^{2} d x .
\end{aligned}
$$

By using the bound $|u| \leq 1,(2.20)$ and the assumption on the support of $\chi_{R, b}$ in $(2.19)$, it is easy to check that,

$$
F_{b, Q_{R}}^{+1}\left(u_{R, b}\right) \leq F_{b, Q_{R}}^{+1}(u)+C R b^{\frac{1}{2}} .
$$

Using (2.21) and (2.18), we get

$$
e_{D}(b, R) \leq e_{N}(b, R)+C R b^{\frac{1}{2}} .
$$

Corollary 2.3. With $\hat{f}(b)$ introduced in 2.10$)$, it holds,

$$
\hat{f}(b)=\lim _{R \longrightarrow+\infty} \frac{e_{N}(b, R)}{R^{2}} .
$$

Proof. We have from 2.7) and 2.17) that, for any $b \in(0,1)$,

$$
e_{D}(b, R)-C R b^{\frac{1}{2}} \leq e_{N}(b, R) \leq e_{D}(b, R) .
$$

Having in mind (2.10), we divide all sides of this inequality by $R^{2}$ and then take the limit as $R \longrightarrow+\infty$. That gives us

$$
\hat{f}(b)=\lim _{R \longrightarrow \infty} \frac{e_{N}(b, R)}{R^{2}} .
$$


Proposition 2.4 (Fournais). There exists a positive constant $C$, such that if 2.16 is satisfied, then

$$
\begin{array}{r}
\frac{e_{D}(b, R)}{R^{2}} \leq \hat{f}(b)+C \frac{\sqrt{b}}{R}, \\
\frac{e_{D}(b, R)}{R^{2}} \geq \hat{f}(b) .
\end{array}
$$

Proof. We have already seen that

$$
\hat{f}(b)=\lim _{R \rightarrow+\infty} \frac{e_{D}(b, R)}{R^{2}} .
$$

Let us first prove 2.28). Let $n \in \mathbb{N}^{*}$ and $R>0$. Let $u \in H_{0}^{1}\left(Q_{R}\right)$ be a minimizer of $F_{b, Q_{R}}^{+1}$ (i.e. $\left.e_{D}(b, R)=F_{b, Q_{R}}^{+1}(u)\right)$. We extend $u$ to a function $\widetilde{u} \in H_{0}^{1}\left(Q_{n R}\right)$ by 'magnetic periodicity' as follows

$$
\widetilde{u}\left(x_{1}+R, x_{2}\right)=e^{i R \frac{x_{2}}{2}} u\left(x_{1}, x_{2}\right), \quad \widetilde{u}\left(x_{1}, x_{2}+R\right)=e^{-i R \frac{x_{1}}{2}} u\left(x_{1}, x_{2}\right) .
$$

Let $\mathcal{J}^{n}=\left\{j \in \mathbb{Z}, 1 \leq j \leq n^{2}\right\}$. Notice that, the square $Q_{n R}$ is formed exactly of $n^{2}$ squares $\left(\overline{Q_{R}\left(x_{0}^{j}\right)}\right)_{j \in \mathcal{J}^{n}}$. We define in each $Q_{R}\left(x_{0}^{j}\right)$ the following function

$$
u_{j}=\widetilde{u}_{Q_{R}\left(x_{0}^{j}\right)} .
$$

Observe that $u_{j}$ is a minimizer of $F_{b, Q_{R}\left(x_{0}^{j}\right)}^{+1}$ in $H_{0}^{1}\left(Q_{R}\left(x_{0}^{j}\right)\right)$ and if we extend $u_{j}$ by 0 outside of $Q_{R}\left(x_{0}^{j}\right)$, keeping the same notation $u_{j}$ for this extension, we have, $\widetilde{u}=\sum_{i \in \mathcal{J}^{n}} u_{j}$. Using magnetic translation invariance, it is easy to check that

$$
F_{b, Q_{n R}}^{+1}(\widetilde{u})=\sum_{j \in \mathcal{J}^{n}} F_{b, Q_{R}}^{+1}\left(u_{j}\right)=n^{2} e_{D}(b, R) .
$$

Consequently, we get

$$
e_{D}(b, n R) \leq n^{2} e_{D}(b, R) .
$$

We now divide both sides of this inequality by $n^{2} R^{2}$ then we take the limit as $n \longrightarrow \infty$. Having in mind 2.10, this gives 2.28).

We prove (2.27).

If $n \in \mathbb{N}^{*}$ and $j=\left(j_{1}, j_{2}\right) \in \mathbb{Z}^{2}$, we denote by

$$
K_{j}=I_{j_{1}} \times I_{j_{2}},
$$

where

$$
\forall m \in \mathbb{Z}, \quad I_{m}=\left(\frac{2 m+1-n}{2}-\frac{1}{2}, \frac{2 m+1-n}{2}+\frac{1}{2}\right) .
$$

For all $R>0$, we set

$$
Q_{R, j}=\left\{R x: x \in K_{j}\right\} .
$$

Let $\mathcal{J}^{n}=\left\{j=\left(j_{1}, j_{2}\right) \in \mathbb{Z}^{2}: 0 \leq j_{1}, j_{2} \leq n-1\right\}$ and $Q_{n R}=\left(-\frac{n R}{2}, \frac{n R}{2}\right) \times\left(-\frac{n R}{2}, \frac{n R}{2}\right)$. Then the family $\left(\bar{Q}_{R, j}\right)$ is a covering of $Q_{n R}$, formed exactly of $n^{2}$ squares. Let $u=u_{n R} \in H_{0}^{1}\left(Q_{n R}\right)$ be a minimizer of of $F_{b, Q_{n R}}^{+1}$ i.e. $F_{b, Q_{n R}}^{+1}(u)=e_{D}(b, n R)$. We have the obvious decomposition,

$$
\int_{Q_{n R}}|u(x)|^{4} d x=\sum_{i \in \mathcal{J}^{n}} \int_{Q_{R, j}}|u(x)|^{4} d x .
$$

Let $\chi=\chi_{R, b^{\frac{1}{2}}}\left(x-x_{0}^{j}\right)$, where $\chi_{R, b^{\frac{1}{2}}}$ is the cut-off function introduced in 2.19). The function $u$ satisfies $-b\left(\nabla-i \mathbf{A}_{0}\right)^{2} u=\left(1-|u|^{2}\right) u$ in $Q_{n R}$. It results from an integration by parts that

$$
e_{D}(b, n R)=F_{b, Q_{n R}}^{+1}(u)=-\frac{1}{2} \int_{Q_{n R}}\left(|u(x)|^{4}-1\right) d x .
$$


We may write,

$$
\begin{aligned}
\int_{Q_{R, j}}\left|\left(\nabla-i \mathbf{A}_{0}\right) \chi u\right|^{2} d x & =\left\langle\left(\nabla-i \mathbf{A}_{0}\right) \chi u,\left(\nabla-i \mathbf{A}_{0}\right) \chi u\right\rangle \\
& =\langle\nabla \chi u, \nabla \chi u\rangle+\left\langle\chi\left(\nabla-i \mathbf{A}_{0}\right) u, \chi\left(\nabla-i \mathbf{A}_{0}\right) u\right\rangle \\
& +2\left\langle\nabla \chi u, \chi\left(\nabla-i \mathbf{A}_{0}\right) u\right\rangle \\
& =\langle\nabla \chi u, \nabla \chi u\rangle+\left\langle\left(\nabla-i \mathbf{A}_{0}\right)\left(\chi^{2} u\right),\left(\nabla-i \mathbf{A}_{0}\right) u\right\rangle
\end{aligned}
$$

An integration by parts gives us

$$
\int_{Q_{R, j}}\left|\left(\nabla-i \mathbf{A}_{0}\right) \chi u\right|^{2} d x=\int_{Q_{R, j}}|\nabla \chi|^{2}|u|^{2} d x-\left\langle\chi^{2} u,\left(\nabla-i \mathbf{A}_{0}\right)^{2} u\right\rangle .
$$

Using (2.32), we may express the energy $F_{b, Q_{R, j}}^{+1}(\chi u)$ as follows:

$$
\begin{aligned}
F_{b, Q_{R, j}}^{+1}(\chi u) & =\int_{Q_{R, j}}\left(b\left|\left(\nabla-i \mathbf{A}_{0}\right) \chi u\right|^{2}-|\chi u|^{2}\right) d x+\frac{1}{2} \int_{Q_{R, j}}\left(|\chi u|^{4}+1\right) d x \\
& =-\left\langle\chi^{2} u,\left(b\left(\nabla-i \mathbf{A}_{0}\right)^{2}+1\right) u\right\rangle+b \int_{Q_{R, j}}|\nabla \chi|^{2}|u|^{2} d x+\frac{1}{2} \int_{Q_{R, j}}\left(\chi^{4}|u|^{4}+1\right) d x .
\end{aligned}
$$

Using the equation $\left(b\left(\nabla-i \mathbf{A}_{0}\right)^{2}+1\right) u=|u|^{2} u$ and the inequality $\chi^{4} \leq \chi^{2}$, we get

$$
\begin{aligned}
F_{b, Q_{R, j}}^{+1}(\chi u) & \leq b \int_{Q_{R, j}}|\nabla \chi|^{2}|u|^{2} d x-\frac{1}{2} \int_{Q_{R, j}}\left(\chi^{2}|u|^{4}-1\right) d x \\
& \leq b \int_{Q_{R, j}}|\nabla \chi|^{2}|u|^{2} d x+\frac{1}{2} \int_{Q_{R, j}}\left(1-\chi^{2}\right) d x-\frac{1}{2} \int_{Q_{R, j}}\left(|u|^{4}-1\right) d x \\
& \leq-\frac{1}{2} \int_{Q_{R, j}}\left(|u|^{4}-1\right) d x+C b^{\frac{1}{2}} R .
\end{aligned}
$$

Since each $\chi u$ has support in a square of side length $R$, we get

$$
F_{b, Q_{R, j}}^{+1}(\chi u) \geq e_{D}(b, R) .
$$

We sum over the $n^{2}$ squares $\left(Q_{R, j}\right)_{j \in \mathcal{J}^{n}}$ (that cover $Q_{n R}$ ), and get

$$
n^{2} e_{D}(b, R) \leq-\frac{1}{2} \int_{Q_{n R}}\left(|u|^{4}-1\right) d x+C b^{\frac{1}{2}} R n^{2} .
$$

Using (2.31), we obtain

$$
n^{2} e_{D}(b, R) \leq e_{D}(b, n R)+C n^{2} R b^{\frac{1}{2}} .
$$

Dividing by $n^{2} R^{2}$, we obtain

$$
\frac{e_{D}(b, R)}{R^{2}} \leq \frac{e_{D}(b, n R)}{(n R)^{2}}+C R^{-1} b^{\frac{1}{2}}
$$

We take the limit $n \rightarrow+\infty$ and get 2.27).

\section{UPPER BOUND OF THE ENERGY}

The aim of this section is to give an upper bound on the ground state energy $\mathrm{E}_{\mathrm{g}}(\kappa, H)$ introduced in 1.6 .

In the sequel, for some choice of $\rho \in(0,1)$ to be determined later (see (3.10), we consider triples $\left(\ell, x_{0}, \widetilde{x}_{0}\right)$ such that $\overline{Q_{\ell}\left(x_{0}\right)} \subset\left\{\left|B_{0}\right|>\rho\right\} \cap \Omega$ and $\widetilde{x}_{0} \in \overline{Q_{\ell}\left(x_{0}\right)}$. In this situation, we say that this 
triple is $\rho$-admissible, that the pair $\left(\ell, x_{0}\right)$ is $\rho$-admissible and the corresponding square $Q_{\ell}\left(x_{0}\right)$ is a $\rho$-admissiblle. Let us introduce the function:

$$
w_{\ell, x_{0}, \widetilde{x}_{0}}(x)= \begin{cases}e^{i \kappa H \varphi_{x_{0}, \widetilde{x}_{0}}} u_{R}\left(\frac{R}{\ell}\left(x-x_{0}\right)\right) & \text { if } x \in Q_{\ell}\left(x_{0}\right) \subset\left\{B_{0}>\rho\right\} \cap \Omega \\ e^{i \kappa H \varphi_{x_{0}}, \widetilde{x}_{0}} \bar{u}_{R}\left(\frac{R}{\ell}\left(x-x_{0}\right)\right) & \text { if } x \in Q_{\ell}\left(x_{0}\right) \subset\left\{B_{0}<-\rho\right\} \cap \Omega,\end{cases}
$$

where $u_{R} \in H_{0}^{1}(\Omega)$ is a minimizer of the functional in 2.1 and $\varphi_{x_{0}, \widetilde{x}_{0}}$ is the function introduced in [2, Lemma A.3] that satisfies

$$
\left|\mathbf{F}(x)-\sigma_{\ell}\right| B_{0}\left(\widetilde{x}_{0}\right)\left|\mathbf{A}_{0}\left(x-x_{0}\right)-\nabla \varphi_{x_{0}, \widetilde{x}_{0}}(x)\right| \leq C \ell^{2}, \quad\left(x \in Q_{\ell}\left(x_{0}\right)\right),
$$

where $B_{0}\left(\widetilde{x}_{0}\right)=\operatorname{curl} \mathbf{F}\left(\widetilde{x}_{0}\right), \mathbf{A}_{0}$ is the magnetic potential introduced in $(2.2)$ and $\sigma_{\ell}$ is the sign of $B_{0}(x)$ in $Q_{\ell}\left(x_{0}\right)$.

Proposition 3.1. Under Assumption (1.2), there exist positive constants $C$ and $\kappa_{0}$ such that if $\kappa \geq \kappa_{0}, \ell \in(0,1), \delta \in(0,1), \rho>0, \ell^{2} \kappa H \rho>1$, and $\left(\ell, x_{0}, \widetilde{x}_{0}\right)$ is a $\rho$-admissible triple, then,

$$
\frac{1}{\left|Q_{\ell}\left(x_{0}\right)\right|} \mathcal{E}_{0}\left(w_{\ell, x_{0}, \widetilde{x}_{0}}, \mathbf{F}, Q_{\ell}\left(x_{0}\right)\right) \leq(1+\delta) \kappa^{2} \hat{f}\left(\frac{H}{\kappa}\left|B_{0}\left(\widetilde{x}_{0}\right)\right|\right)+C\left(\frac{1}{\ell H}+\delta^{-1} \ell^{4} \kappa H\right) \kappa H .
$$

Proof. Let

$$
R=\ell \sqrt{\kappa H\left|B_{0}\left(\widetilde{x}_{0}\right)\right|} \quad \text { and } \quad b=\frac{H}{\kappa}\left|B_{0}\left(\widetilde{x}_{0}\right)\right| .
$$

We estimate $\mathcal{E}_{0}\left(w_{\ell, x_{0}, \widetilde{x}_{0}}, \mathbf{F}, Q_{\ell}\left(x_{0}\right)\right)$ from above. We write for any $\delta \in(0,1)$

$$
\begin{aligned}
\mathcal{E}_{0}\left(w_{\ell, x_{0}, \widetilde{x}_{0}}, \mathbf{F}, Q_{\ell}\left(x_{0}\right)\right) & \\
= & \int_{Q_{\ell}\left(x_{0}\right)}\left[\left|(\nabla-i \kappa H \mathbf{F}) w_{\ell, x_{0}, \widetilde{x}_{0}}\right|^{2}+\frac{\kappa^{2}}{2}\left(1-\left|w_{\ell, x_{0}, \widetilde{x}_{0}}\right|^{2}\right)^{2}\right] d x \\
\leq & (1+\delta) \int_{Q_{\ell}\left(x_{0}\right)}\left[\left|\left(\nabla-i \kappa H\left(\sigma_{\ell}\left|B_{0}\left(\widetilde{x}_{0}\right)\right| \mathbf{A}_{0}\left(x-x_{0}\right)+\nabla \varphi_{x_{0}, \widetilde{x}_{0}}(x)\right)\right) w_{\ell, x_{0}, \widetilde{x}_{0}}\right|^{2}\right. \\
& \left.+\frac{\kappa^{2}}{2}\left(1-\left|w_{\ell, x_{0}, \widetilde{x}_{0}}\right|^{2}\right)^{2}\right] d x \\
& +C(\kappa H)^{2} \delta^{-1} \int_{Q_{\ell}\left(x_{0}\right)}\left|\mathbf{F}-\left(\sigma_{\ell}\left|B_{0}\left(\widetilde{x}_{0}\right)\right| \mathbf{A}_{0}\left(x-x_{0}\right)+\nabla \varphi_{x_{0}, \widetilde{x}_{0}}(x)\right) w_{\ell, x_{0}, \widetilde{x}_{0}}\right|^{2} d x \\
\leq & (1+\delta) \mathcal{E}_{0}\left(w_{\ell, x_{0}, \widetilde{x}_{0}}, \sigma_{\ell}\left|B_{0}\left(\widetilde{x}_{0}\right)\right| \mathbf{A}_{0}\left(x-x_{0}\right)+\nabla \varphi_{x_{0}, \widetilde{x}_{0}}(x), Q_{\ell}\left(x_{0}\right)\right)+C \delta^{-1} \ell^{6}(\kappa H)^{2} .
\end{aligned}
$$

Using (2.9), the definition of $w_{\ell, x_{0}, \widetilde{x}_{0}}$ and the change of variable $y=\frac{R}{\ell}\left(x-x_{0}\right)$, we obtain

$$
\begin{aligned}
& \mathcal{E}_{0}\left(w_{\ell, x_{0}, \widetilde{x}_{0}}, \sigma_{\ell}\left|B_{0}\left(\widetilde{x}_{0}\right)\right| \mathbf{A}_{0}\left(x-x_{0}\right)+\nabla \varphi_{x_{0}, \widetilde{x}_{0}}(x), Q_{\ell}\left(x_{0}\right)\right) \\
& =\int_{Q_{R}}\left[\left|\left(\frac{R}{\ell} \nabla_{y}-i \frac{R}{\ell} \mathbf{A}_{0}(y)\right) u_{R}(y)\right|^{2}+\frac{\kappa^{2}}{2}\left(1-\left|u_{R}(y)\right|^{2}\right)^{2}\right] \frac{\ell^{2}}{R^{2}} d y \\
& =\frac{1}{b} F_{b, Q_{R}}^{+1}\left(u_{R}\right) .
\end{aligned}
$$

Since $u_{R} \in H_{0}^{1}\left(Q_{R}\right)$ is a minimizer of $F_{b, Q_{R}}^{+1}$, then

$$
F_{b, Q_{R}}^{+1}\left(u_{R}\right)=e_{D}(b, R) .
$$

Proposition 2.4 tells us that $\frac{e_{D}(b, R)}{R^{2}} \leq \hat{f}(b)+C \frac{\sqrt{b}}{R}$ for all $\left.b \in\right] 0,1[$ and $R \geq 1$. This assumption is satisfied because $R \geq \ell \sqrt{\kappa H \rho}>1$ (see Remark 3.2). Therefore, we get from (3.6) and (3.7) 
the estimate

$$
\mathcal{E}_{0}\left(w_{\ell, x_{0}, \widetilde{x}_{0}}, \sigma_{\ell}\left|B_{0}\left(\widetilde{x}_{0}\right)\right| \mathbf{A}_{0}\left(x-x_{0}\right)+\nabla \varphi_{x_{0}, \widetilde{x}_{0}}(x), Q_{\ell}\left(x_{0}\right)\right) \leq R^{2} \frac{\hat{f}(b)}{b}+C \frac{R}{\sqrt{b}},
$$

with $b$ defined in (3.4).

We get by collecting the estimates in (3.5)-(3.6) that,

$$
\mathcal{E}_{0}\left(w_{\ell, x_{0}, \widetilde{x}_{0}}, \mathbf{F}, Q_{\ell}\left(x_{0}\right)\right) \leq(1+\delta) R^{2} \frac{\hat{f}(b)}{b}+C_{1} \frac{R}{\sqrt{b}}+C_{2} \delta^{-1} \ell^{6}(\kappa H)^{2} .
$$

Remembering the definition of $b$ and $R$ in (3.4), we get

$$
\frac{1}{\left|Q_{\ell}\left(x_{0}\right)\right|} \mathcal{E}_{0}\left(w_{\ell, x_{0}, \widetilde{x}_{0}}, \mathbf{F}, Q_{\ell}\left(x_{0}\right)\right) \leq(1+\delta) \kappa^{2} \hat{f}\left(\frac{H}{\kappa}\left|B_{0}\left(\widetilde{x}_{0}\right)\right|\right)+C\left(\frac{\kappa}{\ell}+\delta^{-1} \ell^{4}(\kappa H)^{2}\right),
$$

which finishes the proof of Proposition 3.1 .

Remark 3.2. We select $\ell, \delta$ and $\rho$ as follow:

$$
\ell=(\kappa H)^{-\frac{1}{4}}, \quad \rho=(\kappa H)^{-\frac{1}{3}} .
$$

and

$$
\delta=\left(\ln \frac{\kappa}{H}\right)^{-\frac{1}{4}}
$$

Under Assumption (1.7), this choice permits us to verify the assumptions in Proposition 3.1 and to obtain error terms of order $o\left(\kappa H \ln \frac{\kappa}{H}\right)$. We have indeed as $\kappa \longrightarrow+\infty$

$$
\begin{gathered}
\frac{\kappa}{\ell \kappa H \ln \frac{\kappa}{H}}=\frac{\kappa^{\frac{1}{4}}}{H^{\frac{3}{4}} \ln \frac{\kappa}{H}} \ll 1, \\
\frac{\delta^{-1}(\kappa H)^{2} \ell^{4}}{\kappa H \ln \frac{\kappa}{H}}=\frac{1}{\left(\ln \frac{\kappa}{H}\right)^{\frac{3}{4}}} \ll 1, \\
\ell(\kappa H)^{\frac{1}{2}} \rho^{\frac{1}{2}}=(\kappa H)^{\frac{1}{12}} \gg 1 .
\end{gathered}
$$

Theorem 3.3. Under Assumption (1.2), if (1.7) holds, then, the ground state energy $\mathrm{E}_{\mathrm{g}}(\kappa, H)$ in (1.6) satisfies

$$
\mathrm{E}_{\mathrm{g}}(\kappa, H) \leq \kappa^{2} \int_{\Omega} \hat{f}\left(\frac{H}{\kappa}\left|B_{0}(x)\right|\right) d x+o\left(\kappa H \ln \frac{\kappa}{H}\right), \quad \text { as } \kappa \longrightarrow+\infty .
$$

Proof. Let $\ell \in(0,1), \delta$ and $\rho$ be the parameters depending on $\kappa$ and chosen as in Remark 3.2 . As we did in the previous paper [2, Proposition 5.1], we consider the lattice $\Gamma_{\ell}:=\ell \mathbb{Z} \times \ell \mathbb{Z}$ and write, for $\gamma, \widetilde{\gamma} \in \Gamma_{\ell}$,

$$
Q_{\gamma, \ell}=Q_{\ell}(\gamma) \text { and } w_{\ell, x_{0}, \widetilde{x}_{0}}=w_{\ell, \gamma, \widetilde{\gamma}}
$$

For any $\gamma \in \Gamma_{\ell}$ such that $Q_{\gamma, \ell}$ is $\rho$-admissible square, let

$$
\underline{B}_{\gamma, \ell}=\inf _{x \in Q_{\gamma, \ell}}\left|B_{0}(x)\right|
$$

and

$$
\mathcal{I}_{\ell, \rho}=\left\{\gamma ; \overline{Q_{\gamma, \ell}} \subset \Omega \cap\left\{\left|B_{0}\right|>\rho\right\}\right\}, \quad N=\operatorname{card} \mathcal{I}_{\ell, \rho} .
$$

Then as $\kappa \rightarrow+\infty$, we have:

$$
N=|\Omega| \ell^{-2}+\mathcal{O}\left(\ell^{-1}\right)+\mathcal{O}\left(\rho \ell^{-2}\right)
$$

For all $x \in \Omega$, we define,

$$
s(x)=\sum_{\gamma \in \mathcal{J}_{\ell, \rho}} w_{\ell, \gamma, \widetilde{\gamma}}(x)
$$


where $w_{\ell, \gamma, \widetilde{\gamma}}$ has been extended by 0 outside of $Q_{\gamma, \ell}$. Remember the functional $\mathcal{E}_{\kappa, H}$ in $(1.1)$. We compute the energy of the test configuration $(s, \mathbf{F})$. Since $\operatorname{curl} \mathbf{F}=B$, we get,

$$
\mathcal{E}_{\kappa, H}(s, \mathbf{F}, \Omega)=\sum_{\gamma \in \mathcal{J}_{\ell, \rho}} \mathcal{E}_{0}\left(w_{\ell, \gamma, \widetilde{\gamma}}, \mathbf{F}, Q_{\gamma, \ell}\right)
$$

Recall that for any $\widetilde{\gamma} \in Q_{\gamma, \ell}, B_{0}(\widetilde{\gamma})$ satisfies 3.2$)$. Then, we select $\widetilde{\gamma} \in Q_{\gamma, \ell}$ such that

$$
\left|B_{0}(\widetilde{\gamma})\right|=\underline{B}_{\gamma, \ell} .
$$

Using Proposition 3.1 and noticing that $\left|Q_{\gamma, \ell}\right|=\ell^{2}$, we get for any $\delta \in(0,1)$

$$
\sum_{\gamma \in \mathcal{J}_{\ell, \rho}} \mathcal{E}_{0}\left(w_{\ell, \gamma, \widetilde{\gamma}}, \mathbf{F}, Q_{\gamma, \ell}\right) \leq \kappa^{2}(1+\delta) \sum_{\gamma \in \mathcal{J}_{\ell, \rho}} \hat{f}\left(\frac{H}{\kappa} \underline{B}_{\gamma, \ell}\right) \ell^{2}+r(\kappa, H, \ell),
$$

where

$$
r(\kappa, H, \ell)=\mathcal{O}\left(\frac{\kappa}{\ell}+\delta^{-1} \ell^{4}(\kappa H)^{2}\right) .
$$

Having in mind Property (3) of the function $\hat{f}$ established in Theorem 2.1, we recognize the lower Riemann sum and notice that $\cup_{\gamma \in \mathcal{J}_{\ell, \rho}} Q_{\gamma, \ell} \subset \Omega$, then, we get by collecting (3.17)-3.18) that

$$
\mathcal{E}_{\kappa, H}(s, \mathbf{F}, \Omega) \leq(1+\delta) \kappa^{2} \int_{\Omega} \hat{f}\left(\frac{H}{\kappa}\left|B_{0}(x)\right|\right) d x+r(\kappa, H, \ell) .
$$

The choice of the parameters $\delta$ in (3.11) and $\ell$ in 3.10 implies that all error terms are of lower order compared to $\kappa H \ln \frac{\kappa}{H}$.

Remark 3.4. The remainder term in 3.19 is small compared with the leading order term. We have, for any $\rho_{0}>0$

$$
\begin{aligned}
\kappa^{2} \int_{\Omega} \hat{f}\left(\frac{H}{\kappa}\left|B_{0}(x)\right|\right) d x & \geq \kappa^{2} \int_{\Omega \cap\left\{\left|B_{0}\right|>\rho_{0}\right\}} \hat{f}\left(\frac{H}{\kappa}\left|B_{0}(x)\right|\right) d x \\
& \geq \kappa^{2} \hat{f}\left(\frac{H}{\kappa} \rho_{0}\right)\left|\Omega \cap\left\{\left|B_{0}\right|>\rho_{0}\right\}\right| .
\end{aligned}
$$

In view of 2.11), for all positive constant $C$ there exists $\rho_{0}>0$ such that if $H \leq C \kappa$ and $\frac{\rho_{0}}{C_{1}}$ is sufficiently small for some $C_{1}>0$, then as $\kappa \longrightarrow+\infty$

$$
\kappa^{2} \int_{\Omega} \hat{f}\left(\frac{H}{\kappa}\left|B_{0}(x)\right|\right) d x \geq C_{2} \frac{\kappa H \rho_{0}}{2} \ln \frac{\kappa}{H \rho_{0}}(1+o(1)),
$$

where $C_{2}$ is a positive constant.

In particular, when (1.7) is satisfied, we see that,

$$
r(\kappa, H, \ell) \ll \kappa^{2} \int_{\Omega} \hat{f}\left(\frac{H}{\kappa}\left|B_{0}(x)\right|\right) d x .
$$

\section{A PRIORI ESTIMATES OF MINIMIZERS}

The aim of this section is to give a priori estimates on the solutions of the Ginzburg-Landau equations 1.5. These estimates play an essential role in controlling the error resulting from various approximations. The starting point is the following $L^{\infty}$-bound resulting from the maximum principle. If $(\psi, \mathbf{A}) \in H^{1}(\Omega ; \mathbb{C}) \times H_{\text {div }}^{1}\left(\mathbb{R}^{2}\right)$ is a solution of 1.5$)$, then

$$
\|\psi\|_{L^{\infty}(\Omega)} \leq 1 .
$$

Next we prove an estimate on the induced magnetic potential. 
Proposition 4.1. Suppose that the magnetic field $H$ is a function of $\kappa$ and satisfies (1.7). Let $\alpha \in(0,1)$. There exist positive constants $\kappa_{0}$ and $C$ such that, if $\kappa \geq \kappa_{0}$ and $(\psi, \mathbf{A})$ is a minimizer of (1.1), then

$$
\begin{gathered}
\|\mathbf{A}-\mathbf{F}\|_{H^{2}(\Omega)} \leq \frac{C}{H}\left(\int_{\Omega} \hat{f}\left(\frac{H}{\kappa}\left|B_{0}(x)\right|\right) d x\right)^{\frac{1}{2}}, \\
\|\mathbf{A}-\mathbf{F}\|_{C^{0, \alpha}(\bar{\Omega})} \leq \frac{C}{H}\left(\int_{\Omega} \hat{f}\left(\frac{H}{\kappa}\left|B_{0}(x)\right|\right) d x\right)^{\frac{1}{2}} .
\end{gathered}
$$

Here $\mathbf{F}$ is the magnetic potential introduced in 1.3 .

Proof. The estimate in $C^{0, \alpha}$-norm is a consequence of the continuous Sobolev embedding of $H^{2}(\Omega)$ in $C^{0, \alpha}(\bar{\Omega})$.

It is easy to show that

$$
\|\operatorname{curl}(\mathbf{A}-\mathbf{F})\|_{L^{2}(\Omega)} \leq \frac{1}{\kappa H} \mathrm{E}_{\mathrm{g}}(\kappa, H)^{\frac{1}{2}}
$$

and

$$
\|(\nabla-i \kappa H \mathbf{A}) \psi\|_{L^{2}(\Omega)} \leq \mathrm{E}_{\mathrm{g}}(\kappa, H)^{\frac{1}{2}} .
$$

Notice that under Assumption (1.7), it follows from Theorem 3.3 and Remark 3.4 that

$$
\begin{aligned}
& \|\operatorname{curl}(\mathbf{A}-\mathbf{F})\|_{L^{2}(\Omega)} \leq \frac{1}{H}\left(\int_{\Omega} \hat{f}\left(\frac{H}{\kappa}\left|B_{0}(x)\right|\right) d x\right)^{\frac{1}{2}}, \\
& \|(\nabla-i \kappa H \mathbf{A}) \psi\|_{L^{2}(\Omega)} \leq \kappa\left(\int_{\Omega} \hat{f}\left(\frac{H}{\kappa}\left|B_{0}(x)\right|\right) d x\right)^{\frac{1}{2}} .
\end{aligned}
$$

Let $a=\mathbf{A}-\mathbf{F}$. We will prove that

$$
\|a\|_{H^{2}(\Omega)} \leq \frac{C}{H}\left(\int_{\Omega} \hat{f}\left(\frac{H}{\kappa}\left|B_{0}(x)\right|\right) d x\right)^{\frac{1}{2}} .
$$

Since $\operatorname{div} a=0$ and $a \cdot \nu=0$ on $\partial \Omega$, we get by regularity of the curl-div system see [5. Appen$\operatorname{dix}$ A.5]

$$
\|a\|_{H^{2}(\Omega)} \leq C^{\prime}\|\operatorname{curl} a\|_{H^{1}(\Omega)} .
$$

The second equation in $(1.5)$ reads as follows:

$$
-\nabla^{\perp} \operatorname{curl} a=\frac{1}{\kappa H} \operatorname{Im}(\bar{\psi}(\nabla-i \kappa H \mathbf{A}) \psi) .
$$

The estimates in (4.1) and the bound in 4.6), give us

$$
\|a\|_{H^{2}(\Omega)} \leq C\left(\|\operatorname{curl} a\|_{L^{2}(\Omega)}+\frac{1}{\kappa H}\|(\nabla-i \kappa H \mathbf{A}) \psi\|_{L^{2}(\Omega)}\right) .
$$

Inserting the estimates in 4.4 and 4.5 into this upper bound finishes the proof of the proposition.

\section{Proof of Theorem 1.5. Lower Bound}

In this section, we suppose that $\mathcal{D}$ is an open set with smooth boundary such that $\overline{\mathcal{D}} \subset \Omega$. We will give a lower bound of the energy $\mathcal{E}(\psi, \mathbf{A} ; \mathcal{D})$ introduced in 1.17 , when $(\psi, \mathbf{A})$ is a minimizer of the functional in 1.1 . 


\section{Construction of a gauge transformation:}

Let $\phi_{x_{0}}(x)=\left(\mathbf{A}\left(x_{0}\right)-\mathbf{F}\left(x_{0}\right)\right) \cdot x$, where $\mathbf{F}$ is the magnetic potential introduced in (1.3) and $\left(\ell, x_{0}\right)$ a $\rho$-admissible pair. Choosing $\alpha \in(0,1)$ and using the estimate of $\|\mathbf{A}-\mathbf{F}\|_{C^{0, \alpha}(\Omega)}$ given in Proposition 4.1, we get for all $x \in Q_{\ell}\left(x_{0}\right)$,

$$
\begin{aligned}
\left|\mathbf{A}(x)-\nabla \phi_{x_{0}}-\mathbf{F}(x)\right| & =\left|(\mathbf{A}-\mathbf{F})(x)-(\mathbf{A}-\mathbf{F})\left(x_{0}\right)\right| \\
& \leq\|\mathbf{A}-\mathbf{F}\|_{C^{0, \alpha}(\Omega)}\left|x-x_{0}\right|^{\alpha} \\
& \leq C_{\alpha} \lambda \ell^{\alpha},
\end{aligned}
$$

where

$$
\lambda=\frac{1}{H}\left(\int_{\Omega} \hat{f}\left(\frac{H}{\kappa}\left|B_{0}(x)\right|\right) d x\right)^{\frac{1}{2}} .
$$

Using (2.11), it is clear that, under condition (1.7)

$$
\lambda^{2}=\mathcal{O}\left(\frac{1}{\kappa H} \ln \frac{\kappa}{H}\right)
$$

as $\kappa \longrightarrow+\infty$.

Proposition 5.1. For all $\alpha \in(0,1)$, there exist positive constants $C$ and $\kappa_{0}$ such that if $\kappa \geq \kappa_{0}$, $\ell \in\left(0, \frac{1}{2}\right), \delta \in(0,1), \rho>0, \ell^{2} \kappa H \rho>1,(\psi, \mathbf{A}) \in H^{1}(\Omega ; \mathbb{C}) \times H_{\text {div }}^{1}(\Omega)$ is a minimizer of 1.1 , and $\left(\ell, x_{0}, \widetilde{x}_{0}\right)$ a $\rho$-admissible triple, then,

$\frac{1}{\left|Q_{\ell}\left(x_{0}\right)\right|} \mathcal{E}_{0}\left(\psi, \mathbf{A} ; Q_{\ell}\left(x_{0}\right)\right) \geq(1-\delta) \kappa^{2} \hat{f}\left(\frac{H}{\kappa}\left|B_{0}\left(\widetilde{x}_{0}\right)\right|\right)-C\left(\frac{\kappa}{\ell}+\delta^{-1}(\kappa H)^{2} \ell^{4}+\delta^{-1} \kappa^{2} H^{2} \lambda^{2} \ell^{2 \alpha}\right)$.

Proof. Let $\widetilde{x}_{0} \in \overline{Q_{\ell}\left(x_{0}\right)}$. Recall the function $\varphi_{x_{0}, \widetilde{x}_{0}}$ satisfiying $(3.2)$. For all $x \in Q_{\ell}\left(x_{0}\right)$, let

$$
u(x)=e^{-i \kappa H \varphi} \psi(x),
$$

where $\varphi=\varphi_{x_{0}, \widetilde{x}_{0}}+\phi_{x_{0}}$ and $\phi_{x_{0}}$ is introduced in (5.1).

Estimate of $\mathcal{E}_{0}$ in $Q_{\ell}\left(x_{0}\right)$ :

As we did in [2, Lemma 4.1], we have, for any $\delta \in(0,1)$ and $\alpha \in(0,1)$

$$
\begin{aligned}
\mathcal{E}_{0}\left(\psi, \mathbf{A} ; Q_{\ell}\left(x_{0}\right)\right) \geq(1-\delta) \mathcal{E}_{0}\left(u, \sigma_{\ell}\left|B_{0}\left(\widetilde{x}_{0}\right)\right|\right. & \left.\mathbf{A}_{0}\left(x-x_{0}\right) ; Q_{\ell}\left(x_{0}\right)\right) \\
& -C \delta^{-1}(\kappa H)^{2}\left(\ell^{4}+\lambda^{2} \ell^{2 \alpha}\right) \int_{Q_{\ell}\left(x_{0}\right)}|\psi|^{2} d x
\end{aligned}
$$

Let

$$
R=\ell \sqrt{\kappa H\left|B_{0}\left(\widetilde{x}_{0}\right)\right|} \quad \text { and } \quad b=\frac{H}{\kappa}\left|B_{0}\left(\widetilde{x}_{0}\right)\right| .
$$

Define the function in $Q_{R}$

$$
v_{\ell, x_{0}, \widetilde{x}_{0}}(x)= \begin{cases}u\left(\frac{\ell}{R} x+x_{0}\right) & \text { if } x \in Q_{R} \subset\left\{\left\{B_{0}>\rho\right\} \cap \Omega\right\} \\ \bar{u}\left(\frac{\ell}{R} x+x_{0}\right) & \text { if } x \in Q_{R} \subset\left\{\left\{B_{0}<-\rho\right\} \cap \Omega\right\} .\end{cases}
$$

Using (2.9), and the change of variable $y=\frac{R}{\ell}\left(x-x_{0}\right)$, we get

$$
\mathcal{E}_{0}\left(u, \sigma_{\ell}\left|B_{0}\left(\widetilde{x}_{0}\right)\right| \mathbf{A}_{0}\left(x-x_{0}\right) ; Q_{\ell}\left(x_{0}\right)\right)=\frac{1}{b} F_{b, Q_{R}}^{+1}\left(v_{\ell, x_{0}, \widetilde{x}_{0}}\right) .
$$

Here $F_{b, Q_{R}}^{+1}$ is introduced in 2.1. Since $v_{\ell, x_{0}, \widetilde{x}_{0}} \in H^{1}\left(Q_{R}\right)$, we have

$$
F_{b, Q_{R}}^{+1}\left(v_{\ell, x_{0}, \widetilde{x}_{0}}\right) \geq e_{N}(b, R) .
$$

By collecting (2.17)-(2.27) and the lower bound in (5.9), we get,

$$
F_{b, Q_{R}}^{+1}\left(v_{\ell, x_{0}, \widetilde{x}_{0}}\right) \geq R^{2} \hat{f}(b)-C R b^{\frac{1}{2}} .
$$


As a consequence, (5.8) gives us

$$
\mathcal{E}_{0}\left(u, \sigma_{\ell}\left|B_{0}\left(\widetilde{x}_{0}\right)\right| \mathbf{A}_{0}\left(x-x_{0}\right) ; Q_{\ell}\left(x_{0}\right)\right) \geq R^{2} \frac{\hat{f}(b)}{b}-C \frac{R}{\sqrt{b}} .
$$

with $b$ and $R$ introduced in (5.6).

Inserting (5.11) into (5.5) and using the bound of $\psi$ in (4.1), we get

$$
\mathcal{E}_{0}\left(\psi, \mathbf{A} ; Q_{\ell}\left(x_{0}\right)\right) \geq(1-\delta) R^{2} \frac{\hat{f}(b)}{b}-C \frac{R}{\sqrt{b}}-C^{\prime} \delta^{-1}(\kappa H)^{2}\left(\ell^{4}+\lambda^{2} \ell^{2 \alpha}\right) \ell^{2} .
$$

Having in mind (5.6), we get for any $\alpha \in(0,1)$

$$
\mathcal{E}_{0}\left(\psi, \mathbf{A} ; Q_{\ell}\left(x_{0}\right)\right) \geq\left((1-\delta) \kappa^{2} \hat{f}\left(\frac{H}{\kappa}\left|B_{0}\left(\widetilde{x}_{0}\right)\right|\right)-C\left(\frac{\kappa}{\ell}+\delta^{-1}(\kappa H)^{2} \ell^{4}+\delta^{-1} \kappa^{2} H^{2} \lambda^{2} \ell^{2 \alpha}\right)\right) \ell^{2} .
$$

This finishes the proof of Proposition 5.1 .

Remark 5.2. For any $\alpha \in(0,1)$, we keep the same choice of $\ell, \rho$ as in 3.10 and choose $\delta$ as follows:

$$
\delta=\left(\ln \frac{\kappa}{H}\right)^{-\frac{\alpha}{4}} .
$$

This choice and Assumption (1.7) permit us to have the assumptions of Proposition 5.1 satisfied and make the error terms in its statement of order $o\left(\kappa H \ln \frac{\kappa}{H}\right)$. We have as $\kappa \longrightarrow+\infty$,

$$
\begin{aligned}
& \frac{\kappa}{\ell \kappa H \ln \frac{\kappa}{H}}=\frac{\kappa^{\frac{1}{4}}}{H^{\frac{3}{4}} \ln \frac{\kappa}{H}} \ll 1, \\
& \frac{\delta^{-1}(\kappa H)^{2} \ell^{4}}{\kappa H \ln \frac{\kappa}{H}}=\frac{1}{\left(\ln \frac{\kappa}{H}\right)^{1-\frac{\alpha}{4}}} \ll 1, \\
& \frac{\delta^{-1} \kappa H \ln \frac{C_{0} \kappa}{H} \ell^{2 \alpha}}{\kappa H \ln \frac{\kappa}{H}}=\frac{\ln \frac{C_{0} \kappa}{H}}{\left(\ln \frac{\kappa}{H}\right)^{1-\frac{\alpha}{4}}(\kappa H)^{\frac{\alpha}{2}}} \ll 1, \\
& \ell(\kappa H)^{\frac{1}{2}} \rho^{\frac{1}{2}}=(\kappa H)^{\frac{1}{12}} \gg 1 .
\end{aligned}
$$

Remark 5.3. As a byproduct of the proof, we get also a useful estimate. Using the bound $|\psi| \leq 1$, it results from (5.5):

$$
\begin{aligned}
\frac{(1-\delta)}{\left|Q_{\ell}\left(x_{0}\right)\right|} \mathcal{E}_{0}\left(\psi, \sigma_{\ell}\left|B_{0}\left(\widetilde{x}_{0}\right)\right| \mathbf{A}_{0}\left(x-x_{0}\right)+\nabla \varphi, Q_{\ell}\left(x_{0}\right)\right) \leq \frac{1}{\left|Q_{\ell}\left(x_{0}\right)\right|} & \mathcal{E}_{0}\left(\psi, \mathbf{A}, Q_{\ell}\left(x_{0}\right)\right) \\
& +C \delta^{-1}(\kappa H)^{2}\left(\ell^{4}+\lambda^{2} \ell^{2 \alpha}\right) .
\end{aligned}
$$

Using (5.3) and choosing $\ell, \rho$ as in (3.10) and $\delta$ as in (5.13), we get a function $\hat{r}:(0,+\infty) \longmapsto$ $(0,+\infty)$ satisfying $\lim _{t \rightarrow+\infty} \hat{r}(t)=0$ and

$$
\mathcal{E}_{0}\left(\psi, \sigma_{\ell}\left|B_{0}\left(\widetilde{x}_{0}\right)\right| \mathbf{A}_{0}\left(x-x_{0}\right)+\nabla \varphi, Q_{\ell}\left(x_{0}\right)\right) \leq \mathcal{E}_{0}\left(\psi, \mathbf{A}, Q_{\ell}\left(x_{0}\right)\right)+\ell^{2} \kappa H \ln \frac{\kappa}{H} \hat{r}(\kappa),
$$

for any $\widetilde{x}_{0}$ in $Q_{\ell}\left(x_{0}\right)$.

The next theorem presents the lower bound of the local energy in the domain $\mathcal{D}$ such that $\overline{\mathcal{D}} \subset \Omega$ and we deduce the lower bound of the global energy by replacing $\mathcal{D}$ with $\Omega$.

Theorem 5.4. Under Assumption (1.2), if $H(\kappa)$ satisfies 1.7$],(\psi, \mathbf{A}) \in H^{1}(\Omega, \mathbb{C}) \times H_{\text {div }}^{1}(\Omega)$ is a minimizer of (1.1) and $\overline{\mathcal{D}} \subset \Omega$ is open, then,

$$
\mathcal{E}(\psi, \mathbf{A} ; \mathcal{D}) \geq \kappa^{2} \int_{\mathcal{D}} \hat{f}\left(\frac{H}{\kappa}\left|B_{0}(x)\right|\right) d x+o\left(\kappa H \ln \frac{\kappa}{H}\right), \quad \text { as } \kappa \longrightarrow+\infty .
$$


Proof. The proof is similar to the one of Theorem 3.3 .

Let

$$
\mathcal{D}_{\ell, \rho}=\operatorname{int}\left(\cup_{\gamma \in \mathcal{I}_{\ell, \rho}} \overline{Q_{\gamma, \ell}}\right)
$$

and

$$
\bar{B}_{\gamma, \ell}=\sup _{x \in Q_{\gamma, \ell}}\left|B_{0}(x)\right|,
$$

where $\mathcal{I}_{\ell, \rho}$ was introduced in (3.14).

If $(\psi, \mathbf{A})$ is a minimizer of $(1.1)$, we have

$$
\mathcal{E}(\psi, \mathbf{A} ; \mathcal{D})=\mathcal{E}_{0}\left(\psi, \mathbf{A} ; \mathcal{D}_{\ell, \rho}\right)+\mathcal{E}_{0}\left(\psi, \mathbf{A} ; \mathcal{D} \backslash \mathcal{D}_{\ell, \rho}\right)+(\kappa H)^{2} \int_{\Omega}\left|\operatorname{curl} \mathbf{A}-B_{0}\right|^{2} d x,
$$

where $\mathcal{E}_{0}(\psi, \mathbf{A} ; \mathcal{D})$ is introduced in $(1.16)$.

Since the magnetic energy term and the energy in $\mathcal{D} \backslash \mathcal{D}_{\ell, \rho}$ are positive, we may write,

$$
\mathcal{E}(\psi, \mathbf{A} ; \mathcal{D}) \geq \mathcal{E}_{0}\left(\psi, \mathbf{A} ; \mathcal{D}_{\ell, \rho}\right) .
$$

To estimate $\mathcal{E}_{0}\left(\psi, \mathbf{A} ; \mathcal{D}_{\ell, \rho}\right)$, we notice that,

$$
\mathcal{E}_{0}\left(\psi, \mathbf{A} ; \mathcal{D}_{\ell, \rho}\right)=\sum_{\gamma \in \mathcal{I}_{\ell, \rho}} \mathcal{E}_{0}\left(\psi, \mathbf{A} ; Q_{\gamma, \ell}\right) .
$$

Recall that for any $\widetilde{\gamma} \in \overline{Q_{\gamma, \ell}}$ we have $B_{0}(\widetilde{\gamma})$ satisfies $(3.2)$. Then, we select $\widetilde{\gamma}$ such that

$$
\left|B_{0}(\widetilde{\gamma})\right|=\bar{B}_{\gamma, \ell}
$$

Using (5.12), similarly as we did in the upper bound we recognize the upper Riemann sum, and get

$$
\mathcal{E}_{0}\left(\psi, \mathbf{A} ; \mathcal{D}_{\ell, \rho}\right) \geq \kappa^{2}(1-\delta) \int_{\mathcal{D}_{\ell, \rho}} \hat{f}\left(\frac{H}{\kappa}\left|B_{0}(x)\right|\right) d x-C\left(\frac{\kappa}{\ell}+\delta^{-1}(\kappa H)^{2} \ell^{4}+\delta^{-1} \kappa^{2} H^{2} \lambda^{2} \ell^{2 \alpha}\right) .
$$

Notice that using the regularity of $\partial \mathcal{D}$ and $(1.2)$, there exists $C>0$ such that

$$
\forall \ell \in(0,1), \forall \rho \in(0,1), \quad\left|\mathcal{D} \backslash \mathcal{D}_{\ell, \rho}\right| \leq C(\ell+\rho) .
$$

We get by using property (3) of $f$ in Theorem 2.1. Assumption 1.7 and for some choice of $\rho$ to be determined later

$$
\begin{aligned}
\int_{\mathcal{D}_{\ell, \rho}} \hat{f}\left(\frac{H}{\kappa}\left|B_{0}(x)\right|\right) d x & \geq \int_{\mathcal{D}} \hat{f}\left(\frac{H}{\kappa}\left|B_{0}(x)\right|\right) d x-\int_{\mathcal{D} \backslash \mathcal{D}_{\ell, \rho}} \hat{f}\left(\frac{H}{\kappa}\left|B_{0}(x)\right|\right) d x \\
& \geq \int_{\mathcal{D}} \hat{f}\left(\frac{H}{\kappa}\left|B_{0}(x)\right|\right) d x-C \frac{H}{\kappa}\left|\mathcal{D} \backslash \mathcal{D}_{\ell, \rho}\right| .
\end{aligned}
$$

This implies that

$$
\mathrm{E}_{\mathrm{g}}(\kappa, H) \geq \kappa^{2}(1-\delta) \int_{\mathcal{D}} \hat{f}\left(\frac{H}{\kappa}\left|B_{0}(x)\right|\right) d x-r^{\prime}(\kappa, H, \ell),
$$

where

$$
r^{\prime}(\kappa, H, \ell)=\mathcal{O}\left(\kappa H \ell+\kappa H \rho+\frac{\kappa}{\ell}+\delta^{-1}(\kappa H)^{2} \ell^{4}+\delta^{-1} \kappa^{2} H^{2} \lambda^{2} \ell^{2 \alpha}\right) .
$$

Having in mind (5.3), then, the remainder term becomes

$$
r^{\prime}(\kappa, H, \ell)=\mathcal{O}\left(\kappa H \ell+\kappa H \rho+\frac{\kappa}{\ell}+\delta^{-1}(\kappa H)^{2} \ell^{4}+\delta^{-1} \kappa H \ln \frac{C_{0} \kappa}{H} \ell^{2 \alpha}\right) .
$$

The choice of the parameters $\delta$ in (5.13) and $\rho, \ell$ in 3.10 implies all error terms to be of lower order compared with $\kappa H \ln \frac{\kappa}{H}$. This finishes the proof of Theorem 5.4 . 
Remark 5.5. Notice that $\mathcal{E}_{0}(\psi, \mathbf{A} ; \mathcal{D}) \geq \mathcal{E}_{0}\left(\psi, \mathbf{A} ; \mathcal{D}_{\ell, \rho}\right)$. Using 5.19 and 5.21 with the same choices of $\delta, \rho$ and $\ell$ as in Remark 3.2 , we obtain

$$
\mathcal{E}_{0}(\psi, \mathbf{A} ; \mathcal{D}) \geq \kappa^{2} \int_{\mathcal{D}} \hat{f}\left(\frac{H}{\kappa}\left|B_{0}(x)\right|\right) d x+o\left(\kappa H \ln \frac{\kappa}{H}\right) .
$$

Moreover, we can replace $\mathcal{D}$ by $\Omega$ and get

$$
\mathcal{E}_{0}(\psi, \mathbf{A} ; \Omega) \geq \kappa^{2} \int_{\Omega} \hat{f}\left(\frac{H}{\kappa}\left|B_{0}(x)\right|\right) d x+o\left(\kappa H \ln \frac{\kappa}{H}\right) .
$$

\section{Proof of Corollary 1.4}

Having in mind (1.16), we write

$$
\mathcal{E}(\psi, \mathbf{A} ; \Omega)=\mathcal{E}_{0}(\psi, \mathbf{A} ; \Omega)+(\kappa H)^{2} \int_{\Omega}\left|\operatorname{curl} \mathbf{A}-B_{0}\right|^{2} d x .
$$

Using the estimate of $\mathcal{E}(\psi, \mathbf{A} ; \Omega)$ in Theorem 1.1 , we get, as $\kappa \longrightarrow+\infty$

$$
\mathcal{E}_{0}(\psi, \mathbf{A} ; \Omega)+(\kappa H)^{2} \int_{\Omega}\left|\operatorname{curl} \mathbf{A}-B_{0}\right|^{2} d x \leq \kappa^{2} \int_{\Omega} \hat{f}\left(\frac{H}{\kappa}\left|B_{0}(x)\right|\right) d x+o\left(\kappa H \ln \frac{\kappa}{H}\right) .
$$

Remark 5.5 tells us that

$$
\mathcal{E}_{0}(\psi, \mathbf{A} ; \Omega) \geq \kappa^{2} \int_{\Omega} \hat{f}\left(\frac{H}{\kappa}\left|B_{0}(x)\right|\right) d x+o\left(\kappa H \ln \frac{\kappa}{H}\right) .
$$

Therefore, 5.25 becomes

$$
\begin{aligned}
\kappa^{2} \int_{\Omega} \hat{f}\left(\frac{H}{\kappa}\left|B_{0}(x)\right|\right) d x+o\left(\kappa H \ln \frac{\kappa}{H}\right)+ & (\kappa H)^{2} \int_{\Omega}\left|\operatorname{curl} \mathbf{A}-B_{0}\right|^{2} d x \\
& \leq \kappa^{2} \int_{\Omega} \hat{f}\left(\frac{H}{\kappa}\left|B_{0}(x)\right|\right) d x+o\left(\kappa H \ln \frac{\kappa}{H}\right) .
\end{aligned}
$$

By simplification, we get 1.15 .

\section{Proof of Theorem 1.5: upper bound}

One aim of this section is to derive a sharp estimate of $\mathcal{E}_{0}\left(\psi, \mathbf{A} ; Q_{\ell}\left(x_{0}\right)\right)$, when $(\psi, \mathbf{A}) \in$ $H^{1}(\Omega ; \mathbb{C}) \times H_{\text {div }}^{1}(\Omega)$ is a minimizer of $(1.1)$.

The proof of the next proposition is similar to that in [2, Proposition 6.2], by replacing $\frac{1}{R}$ by $\frac{b^{\frac{1}{2}}}{R}$

Proposition 6.1. For $\alpha \in(0,1)$, there exist positive constants $C$ and $\kappa_{0}$ such that if $\kappa \geq \kappa_{0}$, $\ell \in\left(0, \frac{1}{2}\right), \delta \in(0,1), \rho>0, \ell^{2} \kappa H \rho \geq 1,(\psi, \mathbf{A}) \in H^{1}(\Omega ; \mathbb{C}) \times H_{\mathrm{div}}^{1}(\Omega)$ is a minimizer of (1.1), and $\left(\ell, x_{0}, \widetilde{x}_{0}\right)$ a $\rho$-admissible triple, then,

$$
\frac{1}{\left|Q_{\ell}\left(x_{0}\right)\right|} \mathcal{E}_{0}\left(\psi, \mathbf{A} ; Q_{\ell}\left(x_{0}\right)\right) \leq(1+\delta) \kappa^{2} \hat{f}\left(\frac{H}{\kappa}\left|B_{0}\left(\widetilde{x}_{0}\right)\right|\right)+C\left(\frac{\kappa}{\ell}+\delta^{-1} \ell^{4} \kappa^{2} H^{2}+\delta^{-1} \kappa^{2} H^{2} \lambda^{2} \ell^{2 \alpha}\right),
$$

where $\lambda$ is introduced in 5.2 .

Remark 6.2. Under Assumption (1.7), with the choices of $\ell, \rho$ in 3.10 and $\delta$ in (5.13), we get that the error terms in 6.1) are of order $\kappa H \ln \frac{\kappa}{H}$ 
Proposition 6.3. For any $\alpha \in(0,1)$, there exist positive constants $\widehat{C}_{\alpha}$ and $\kappa_{0}$ such that if $\kappa \geq \kappa_{0}, H(\kappa)$ satisfies (1.7), $\ell$ is chosen as in (3.10), $\delta$ as in (5.13), $\ell^{2} \kappa H \rho \geq 1,(\psi, \mathbf{A})$ is a minimizer of (1.1), and $\left(\ell, x_{0}, \widetilde{x}_{0}\right)$ a $\rho$-admissible triple, then

$$
\begin{aligned}
\mid \frac{1}{\left|Q_{\ell}\left(x_{0}\right)\right|} \mathcal{E}_{0}\left(\psi, \sigma_{\ell}\left|B_{0}\left(\widetilde{x}_{0}\right)\right| \mathbf{A}_{0}\left(x-x_{0}\right)+\nabla\left(\varphi_{x_{0}, \widetilde{x}_{0}}+\phi_{x_{0}}\right), Q_{\ell}\left(x_{0}\right)\right) & -\kappa^{2} \hat{f}\left(\frac{H}{\kappa}\left|B_{0}\left(\widetilde{x}_{0}\right)\right|\right) \mid \\
& \leq \widehat{C}_{\alpha} \kappa H\left(\ln \frac{\kappa}{H}\right)^{\frac{\alpha}{4}}
\end{aligned}
$$

where $\mathbf{A}_{0}$ is the magnetic potential introduced in (2.2), $\sigma_{\ell}$ denotes the sign of $B_{0}, \phi_{x_{0}}$ is defined in (5.1) and $\varphi_{x_{0}, \widetilde{x}_{0}}$ is the function satisfying (3.2).

Proof.

Lower bound: We refer to (5.11) and (5.6). We obtain

$$
\frac{1}{\left|Q_{\ell}\left(x_{0}\right)\right|} \mathcal{E}_{0}\left(\psi, \sigma_{\ell}\left|B_{0}\left(\widetilde{x}_{0}\right)\right| \mathbf{A}_{0}\left(x-x_{0}\right)+\nabla\left(\varphi_{x_{0}, \widetilde{x}_{0}}+\phi_{x_{0}}\right), Q_{\ell}\left(x_{0}\right)\right) \geq \kappa^{2} \hat{f}\left(\frac{H\left|B_{0}\left(\widetilde{x}_{0}\right)\right|}{\kappa}\right)-C \frac{\kappa}{\ell},
$$

where $C$ is a positive constant.

If we select $\ell$ as in $(3.10)$, we get

$$
\frac{1}{\left|Q_{\ell}\left(x_{0}\right)\right|} \mathcal{E}_{0}\left(\psi, \sigma_{\ell}\left|B_{0}\left(\widetilde{x}_{0}\right)\right| \mathbf{A}_{0}\left(x-x_{0}\right)+\nabla\left(\varphi_{x_{0}, \widetilde{x}_{0}}+\phi_{x_{0}}\right), Q_{\ell}\left(x_{0}\right)\right) \geq \kappa^{2} \hat{f}\left(\frac{H\left|B_{0}\left(\widetilde{x}_{0}\right)\right|}{\kappa}\right)-C\left(\kappa^{5} H\right)^{\frac{1}{4}}
$$

Assumption 1.7 permits to verify that the remainder $\left(\kappa^{5} H\right)^{\frac{1}{4}}=\mathcal{O}\left(\kappa H\left(\ln \frac{\kappa}{H}\right)^{\frac{\alpha}{4}}\right)$.

Upper bound: Collecting (5.14) and (6.1), we get for any $\alpha \in(0,1)$, the existence of $C^{\prime}>0$ such that

$$
\begin{array}{r}
\frac{1}{\left|Q_{\ell}\left(x_{0}\right)\right|} \mathcal{E}_{0}\left(\psi, \sigma_{\ell}\left|B_{0}\left(\widetilde{x}_{0}\right)\right| \mathbf{A}_{0}\left(x-x_{0}\right)+\nabla\left(\varphi_{x_{0}, \widetilde{x}_{0}}+\phi_{x_{0}}\right), Q_{\ell}\left(x_{0}\right)\right) \leq \kappa^{2} \hat{f}\left(\frac{H}{\kappa}\left|B_{0}\left(\widetilde{x}_{0}\right)\right|\right) \\
+C^{\prime}\left(\frac{\kappa}{\ell}+\delta^{-1} \ell^{4} \kappa^{2} H^{2}+\delta^{-1} \kappa^{2} H^{2} \lambda^{2} \ell^{2 \alpha}\right)
\end{array}
$$

where $\lambda$ is introduced in 5.2 .

Using (5.3) and selecting $\ell$ as in (3.10) and $\delta$ as in (5.13), we get the existence of a constant $C_{\alpha}$ such that

$$
\begin{aligned}
\frac{1}{\left|Q_{\ell}\left(x_{0}\right)\right|} \mathcal{E}_{0}\left(\psi, \sigma_{\ell}\left|B_{0}\left(\widetilde{x}_{0}\right)\right| \mathbf{A}_{0}\left(x-x_{0}\right)+\nabla\left(\varphi_{x_{0}, \widetilde{x}_{0}}+\phi_{x_{0}}\right), Q_{\ell}\left(x_{0}\right)\right) \leq & \kappa^{2} \hat{f}\left(\frac{H}{\kappa}\left|B_{0}\left(\widetilde{x}_{0}\right)\right|\right) \\
& +C_{\alpha} \kappa H\left(\ln \frac{\kappa}{H}\right)^{\frac{\alpha}{4}} .
\end{aligned}
$$

This achieves the proof of the lemma.

The next lemma will be useful in the proof of Theorem 1.5 .

Lemma 6.4. For any $C_{1}>0$, there exist positive constants $C$ and $\kappa_{0}$ such that if $\ell \in(0,1)$, $\kappa_{0} \leq \kappa$ and $(\psi, \mathbf{A}) \in H^{1}(\Omega ; \mathbb{C}) \times H_{\text {div }}^{1}(\Omega)$ is a solution of $(1.5)$, then

$$
\int_{\mathcal{V}_{\ell}\left(\Gamma, C_{1}\right)}|(\nabla-i \kappa H \mathbf{A}) \psi|^{2} d x \leq C \kappa^{2} \ell\left(1+\frac{1}{\kappa \ell^{\frac{3}{2}}}\right)
$$

where

$$
\mathcal{V}_{\ell}\left(\Gamma, C_{1}\right)=\left\{x \in \Omega: \operatorname{dist}(x, \Gamma) \leq C_{1} \ell \text { and } d(x, \partial \Omega) \geq \frac{\ell}{C_{1}}\right\}
$$


Proof. Using 4.5 and the fact that the range of $\hat{f}$ is the interval $[0,1 / 2]$, we get

$$
\|(\nabla-i \kappa H \mathbf{A}) \psi\|_{L^{2}(\Omega)} \leq C \kappa .
$$

Hence the improvment given by the lemma is when $\frac{1}{C} \kappa^{-2} \leq \ell \leq \ell_{0}$.

Let $C_{2}>C_{1}$ and for $\ell$ small enough we define the following sets $\mathcal{D}_{\ell}^{1}=\mathcal{V}_{\ell}\left(\Gamma, C_{1}\right)$ and $\mathcal{D}_{\ell}^{2}=\mathcal{V}_{\ell}\left(\Gamma, C_{2}\right)$. We can construct a cut-off function $\chi_{\ell} \in C_{c}^{\infty}(\Omega)$ such that

$$
0 \leq \chi_{\ell} \leq 1 \text { in } \mathbb{R}^{2}, \quad \operatorname{supp} \chi_{\ell} \subset \mathcal{D}_{\ell}^{2} \subset \subset \Omega, \quad \chi_{\ell}=1 \text { in } \mathcal{D}_{\ell}^{1} \quad \text { and } \quad\left|\nabla \chi_{\ell}\right| \leq \frac{C}{\ell} \text { in } \mathbb{R}^{2},
$$

where $C$ is a positive constant independent of $\ell$.

The minimizer $\psi$ satisfies

$$
\kappa^{2} \psi\left(1-|\psi|^{2}\right)=-(\nabla-i \kappa H \mathbf{A})^{2} \psi \quad \text { in } \Omega .
$$

We multiply the above equation by $\chi_{\ell} \bar{\psi}$, it results from an integration by parts that

$$
\begin{aligned}
\kappa^{2} \int_{\mathcal{D}_{\ell}^{2}} \chi_{\ell}\left(1-|\psi|^{2}\right)|\psi|^{2} d x & =\int_{\mathcal{D}_{\ell}^{2}}(\nabla-i \kappa H \mathbf{A}) \psi \overline{\chi_{\ell}(\nabla-i \kappa H \mathbf{A}) \psi+\psi \nabla \chi_{\ell}} d x \\
& =\int_{\mathcal{D}_{\ell}^{2}} \chi_{\ell}|(\nabla-i \kappa H \mathbf{A}) \psi|^{2} d x+\int_{\mathcal{D}_{\ell}^{2}} \nabla \chi_{\ell} \bar{\psi}(\nabla-i \kappa H \mathbf{A}) \psi d x .
\end{aligned}
$$

Using Hölder inequality, we have

$$
\left|\int_{\mathcal{D}_{\ell}^{2}} \nabla \chi_{\ell} \bar{\psi}(\nabla-i \kappa H \mathbf{A}) \psi d x\right| \leq\|(\nabla-i \kappa H \mathbf{A}) \psi\|_{L^{2}\left(\Omega, \mathbb{C}^{2}\right)}\left\|\left|\nabla \chi_{\ell}\right| \psi\right\|_{L^{2}\left(\mathcal{D}_{\ell}^{2}\right)} .
$$

Notice that $\left|\mathcal{D}_{\ell}^{2}\right| \leq C^{\prime} \ell$. Using (6.9) and the bound $\|\psi\|_{\infty} \leq 1$, we obtain

$$
\left\|\left|\nabla \chi_{\ell}\right| \psi\right\|_{L^{2}\left(\mathcal{D}_{\ell}^{2}\right)} \leq C^{\prime \prime} \ell^{-\frac{1}{2}}
$$

Putting 6.8 and 6.13) into 6.12), we get

$$
\left|\int_{\mathcal{D}_{\ell}^{2}} \nabla \chi_{\ell} \bar{\psi}(\nabla-i \kappa H \mathbf{A}) \psi d x\right| \leq C \kappa \ell^{-\frac{1}{2}}
$$

and consequently

$$
\int_{\mathcal{D}_{\ell}^{2}} \chi_{\ell}|(\nabla-i \kappa H \mathbf{A}) \psi|^{2} d x \leq \kappa^{2} \int_{\mathcal{D}_{\ell}^{2}} \chi_{\ell}\left(1-|\psi|^{2}\right)|\psi|^{2} d x+C \kappa \ell^{-\frac{1}{2}}
$$

The lemma easily follows from the control of the area of $\mathcal{D}_{\ell}^{2}$ and from observing that $\chi_{\ell}=1$ on $\mathcal{D}_{\ell}^{1}$.

Remark 6.5 . We get a similar estimate by replacing in the lemma $\Gamma$ by the boundary $\partial \mathcal{D}$ of a regular open set $\mathcal{D}$ compactly contained in $\Omega$.

\section{End of the proof of Theorem 1.5 .}

The proof of (1.18) is already obtained in Theorem 5.4. Hence it remains only to give the proof of 1.20 .

We keep the same notation as in (3.13), (3.14) and (5.16). If $(\psi, \mathbf{A})$ is a minimizer of (1.1), we start with 1.17 and write,

$$
\mathcal{E}(\psi, \mathbf{A} ; \mathcal{D})=\mathcal{E}_{0}\left(\psi, \mathbf{A} ; \mathcal{D}_{\ell, \rho}\right)+\mathcal{E}_{0}\left(\psi, \mathbf{A} ; \mathcal{D} \backslash \mathcal{D}_{\ell, \rho}\right)+(\kappa H)^{2} \int_{\Omega}|\operatorname{curl}(\mathbf{A}-\mathbf{F})|^{2} d x .
$$

To estimate $\mathcal{E}_{0}\left(\psi, \mathbf{A} ; \mathcal{D}_{\ell, \rho}\right)$, we notice that,

$$
\mathcal{E}_{0}\left(\psi, \mathbf{A} ; \mathcal{D}_{\ell, \rho}\right)=\sum_{\gamma \in \mathcal{I}_{\ell, \rho}} \mathcal{E}_{0}\left(\psi, \mathbf{A} ; Q_{\gamma, \ell}\right) .
$$


Remark 6.2 tells us that the error terms in 6.1) are of order $\kappa H \ln \frac{\kappa}{H}$. Therfore, using (6.1), we get

$$
\mathcal{E}_{0}\left(\psi, \mathbf{A} ; \mathcal{D}_{\ell, \rho}\right) \leq \kappa^{2} \sum_{\gamma \in \mathcal{I}_{\ell, \rho}} \hat{f}\left(\frac{H}{\kappa}\left|B_{0}(\widetilde{\gamma})\right|\right) \ell^{2}+o\left(\kappa H \ln \frac{\kappa}{H}\right), \quad \text { as } \kappa \longrightarrow+\infty .
$$

We select $\widetilde{\gamma} \in \overline{Q_{\ell}(\gamma)}$ such that $\left|B_{0}(\widetilde{\gamma})\right|=\underline{B}_{\gamma, \ell}$, where $\underline{B}_{\gamma, \ell}$ is defined in (3.13). By monotonicity of $\hat{f}, \hat{f}$ is Riemann-integrable and its integral is larger than any lower Riemann sum. Thus

$$
\mathcal{E}_{0}\left(\psi, \mathbf{A} ; \mathcal{D}_{\ell, \rho}\right) \leq \kappa^{2} \int_{\mathcal{D}_{\ell, \rho}} \hat{f}\left(\frac{H}{\kappa}\left|B_{0}(x)\right|\right) d x+o\left(\kappa H \ln \frac{\kappa}{H}\right), \quad \text { as } \kappa \longrightarrow+\infty .
$$

Moreover, recalling that $\hat{f}$ is a positive function and $\mathcal{D}_{\ell, \rho} \subset \mathcal{D}, 6.17$ becomes

$$
\mathcal{E}_{0}\left(\psi, \mathbf{A} ; \mathcal{D}_{\ell, \rho}\right) \leq \kappa^{2} \int_{\mathcal{D}} \hat{f}\left(\frac{H}{\kappa}\left|B_{0}(x)\right|\right) d x+o\left(\kappa H \ln \frac{\kappa}{H}\right), \quad \text { as } \kappa \longrightarrow+\infty .
$$

For estimating $\mathcal{E}_{0}\left(\psi, \mathbf{A} ; \mathcal{D} \backslash \mathcal{D}_{\ell, \rho}\right)$, we use Lemma 6.4 Remark 6.5 and we keep the same choice of $\ell$ and $\rho$ as in 3.10, which implies $\rho \ll \ell$, we obtain that

$$
\int_{\mathcal{D} \backslash \mathcal{D}_{\ell, \rho}}|(\nabla-i \kappa H \mathbf{A}) \psi|^{2} d x \leq C\left(\kappa^{2} \ell+\kappa \ell^{-\frac{1}{2}}\right) .
$$

Adding the second term in the energy leads to

$$
\mathcal{E}_{0}\left(\psi, \mathbf{A} ; \mathcal{D} \backslash \mathcal{D}_{\ell, \rho}\right) \leq C\left(\kappa^{2} \ell+\kappa \ell^{-\frac{1}{2}}\right) .
$$

The second term in the right hand side is controlled by the first one if

$$
\kappa \ell^{\frac{3}{2}} \gg 1
$$

This is effectively satisfied with our choice of $\ell$ and the condition on $H(\kappa)$.

In order to obtain the term $\kappa^{2} \ell$ in 6.20 comparatively small with $\kappa H \ln \frac{\kappa}{H}$, we need a stronger condition than 1.7 on $H(\kappa)$. In fact, we have

$$
\frac{\kappa^{2} \ell}{\kappa H \ln \frac{\kappa}{H}}=\left(\frac{\kappa^{3}}{H^{5}}\right)^{\frac{1}{4}} \frac{1}{\ln \frac{\kappa}{H}}
$$

and thanks to 1.19 , as $\kappa \longrightarrow+\infty$,

$$
\frac{1}{\ln \frac{\kappa}{H}} \ll 1 \text { and } \frac{\kappa^{3}}{H^{5}} \leq C,
$$

where $C$ is a positive constant.

This implies that

and consequently

$$
\kappa^{2} \ell=o\left(\kappa H \ln \frac{\kappa}{H}\right)
$$

$$
\mathcal{E}_{0}\left(\psi, \mathbf{A} ; \mathcal{D} \backslash \mathcal{D}_{\ell, \rho}\right)=o\left(\kappa H \ln \frac{\kappa}{H}\right) .
$$

Corollary 1.4 tells us that, under Assumption (1.7),

$$
(\kappa H)^{2} \int_{\Omega}\left|\operatorname{curl} \mathbf{A}-B_{0}\right|^{2} d x=o\left(\kappa H \ln \frac{\kappa}{H}\right), \quad \text { as } \kappa \longrightarrow+\infty .
$$

Therefore, by collecting 6.18, 6.21 and 6.22 and inserting then into 6.16), we finish the proof of 1.20. 


\section{Vortices And CONCEntration of the EnERGy}

This section is devoted to the proof of Theorem 1.6. We keep the choice of $\ell$ given in (3.10):

$$
\ell=(\kappa H)^{-\frac{1}{4}},
$$

but we select $\rho$ and $\alpha$ as follows:

$$
\rho=\left(\ln \frac{\kappa}{H}\right)^{-\frac{1}{2}}, \quad \alpha=\frac{1}{2} .
$$

7.1. Energy in a $\rho$-admissible box. If $(\psi, \mathbf{A}) \in H^{1}(\Omega ; \mathbb{C}) \times H_{\text {div }}^{1}(\Omega ; \mathbb{R})$, we consider for all $\rho$-admissible pair $\left(\ell, x_{0}\right)$ the local energy in $Q_{\ell}\left(x_{0}\right)$ :

$$
\mathcal{E}_{0}\left(\psi, \mathbf{A}, Q_{\ell}\left(x_{0}\right)\right)=\int_{Q_{\ell}\left(x_{0}\right)}\left(|(\nabla-i \kappa H \mathbf{A}) \psi|^{2}+\frac{\kappa^{2}}{2}\left(1-|\psi|^{2}\right)^{2}\right) d x
$$

7.2. Division of the square $Q_{\ell}\left(x_{0}\right)$.

Let $H=H(\kappa)$ be a function satisfying (1.7). For reasons that will become clear in Proposition 7.3. we need to divide $Q_{\ell}\left(x_{0}\right)$ into $\mathcal{N}=M^{2}$ disjoint open squares $\left(Q_{\delta(\kappa)}^{j}\right)_{j \in \mathcal{J}}$ such that

$$
Q_{\ell}\left(x_{0}\right)=\cup_{j \in \mathcal{J}} \overline{Q_{\delta(\kappa)}^{j}},
$$

with

$$
M=\left[2^{\frac{7}{8}}(\kappa H)^{\frac{1}{4}}\left(\ln \frac{\kappa}{H}\right)^{-\frac{7}{8}}\right],
$$

where for $t \in \mathbb{R},[t]$ denotes the integer part of $t$.

The side length of theses squares is consequently

$$
\delta(\kappa)=\frac{\ell}{M} \sim 2^{-\frac{7}{8}}(\kappa H)^{-\frac{1}{2}}\left(\ln \frac{\kappa}{H}\right)^{\frac{7}{8}} .
$$

Let us introduced for all $\rho$-admissible triple $\left(\ell, x_{0}, \widetilde{x}_{0}\right)$ the functions $b$ and $R$ by

$$
R\left(\kappa, H, \widetilde{x}_{0}\right)=2^{-\frac{7}{8}}\left(\ln \frac{\kappa}{H}\right)^{\frac{7}{8}}\left|B_{0}\left(\widetilde{x}_{0}\right)\right|^{\frac{1}{2}} \quad \text { and } \quad b\left(\kappa, H, \widetilde{x}_{0}\right)=\frac{H}{\kappa}\left|B_{0}\left(\widetilde{x}_{0}\right)\right| .
$$

Notice that $b\left(\kappa, H, \widetilde{x}_{0}\right)$ and $\frac{1}{R\left(\kappa, H, \widetilde{x}_{0}\right)}$ are uniformly $o(1)$ as $\kappa \longrightarrow+\infty$, in the following sense: For all $\epsilon>0$ there exists $\kappa_{0}>0$ such that $\forall \kappa \geq \kappa_{0}, H$ satisfying (1.7), $\rho$ introduced in (7.1) and any $\rho$-admissible triple $\left(\ell, x_{0}, \widetilde{x}_{0}\right)$

$$
\left|b\left(\kappa, H, \widetilde{x}_{0}\right)\right|+\frac{1}{R\left(\kappa, H, \widetilde{x}_{0}\right)}<\epsilon .
$$

In fact, we have as $\kappa \longrightarrow+\infty$

$$
R\left(\kappa, H, \widetilde{x}_{0}\right) \geq 2^{-\frac{7}{8}}\left(\ln \frac{\kappa}{H}\right)^{\frac{7}{8}} \rho^{\frac{1}{2}} \geq \frac{1}{C}\left(\ln \frac{\kappa}{H}\right)^{\frac{5}{8}} \gg 1 .
$$

Since $B_{0} \in C^{\infty}(\bar{\Omega})$, we have also

$$
0<b\left(\kappa, H, \widetilde{x}_{0}\right) \leq \frac{H}{\kappa} \bar{\beta}_{0} \ll 1
$$

where

$$
\bar{\beta}_{0}=\sup _{x \in \bar{\Omega}}\left|B_{0}(x)\right|
$$

More precisely, let

$$
b\left(\kappa, H, \widetilde{x}_{0}\right)=\hat{b}(\kappa, H, \beta) \quad \text { and } \quad R\left(\kappa, H, \widetilde{x}_{0}\right)=\hat{R}(\kappa, H, \beta),
$$


where $\beta=\left|B_{0}\left(\widetilde{x}_{0}\right)\right|$.

We define the function:

$$
h(\kappa, H)=\max \left(\left(\ln \frac{\kappa}{H}\right)^{-\frac{3}{8}}, \sup _{\bar{\beta}_{0} \geq \beta \geq\left(\ln \frac{\kappa}{H}\right)^{-\frac{1}{2}}} \operatorname{err}(\hat{b}(\kappa, H, \beta), \hat{R}(\kappa, H, \beta))\right),
$$

where $\operatorname{err}(b, R)$ is defined in Proposition 2.13 .

Notice that $h$ satisfies

$$
h(\kappa, H)=o(1), \quad \text { as } \kappa \longrightarrow+\infty .
$$

Next, we will use a method introduced by E. Sandier and S. Serfaty in [11]. We distinguish in the family indexed by $\mathcal{J}$ two types of squares respectively called the 'nice squares' $\left(Q_{\delta(\kappa)}^{j}\right)$ which are indexed in $\mathcal{J}^{n}$ and the 'bad squares' $\left(Q_{\delta(\kappa)}^{j}\right)$ indexed in $\mathcal{J}^{b}$. The set $\mathcal{J}^{n}$ is the set of indices $j \in \mathcal{J}$ such that

$$
\mathcal{E}_{0}\left(\psi, \sigma_{\ell}\left|B_{0}\left(\widetilde{x}_{0}\right)\right| \mathbf{A}_{0}\left(x-x_{0}\right)+\nabla \varphi, Q_{\delta(\kappa)}^{j}\right) \leq \delta(\kappa)^{2} \kappa^{2} \hat{f}\left(\frac{H}{\kappa}\left|B_{0}\left(\widetilde{x}_{0}\right)\right|\right)\left(1+h(\kappa, H)^{\frac{1}{2}}\right) .
$$

The set $\mathcal{J}^{b}$ is the set of indices $j \in \mathcal{J}$ such that

$$
\mathcal{E}_{0}\left(\psi, \sigma_{\ell}\left|B_{0}\left(\widetilde{x}_{0}\right)\right| \mathbf{A}_{0}\left(x-x_{0}\right)+\nabla \varphi, Q_{\delta(\kappa)}^{j}\right)>\delta(\kappa)^{2} \kappa^{2} \hat{f}\left(\frac{H}{\kappa}\left|B_{0}\left(\widetilde{x}_{0}\right)\right|\right)\left(1+h(\kappa, H)^{\frac{1}{2}}\right) .
$$

Hence we have $\mathcal{J}=\mathcal{J}^{n} \cup \mathcal{J}^{b}$. We denote by $\mathcal{N}^{g}$ the cardinal of $\mathcal{J}^{n}$ and by $\mathcal{N}^{b}$ the cardinal of $\mathcal{J}^{b}$.

Lemma 7.1. There exist positive constants $C$ and $\kappa_{0}$ such that if $\kappa \geq \kappa_{0}$, then

$$
\mathcal{N}^{b} \leq C \frac{h(\kappa, H)^{\frac{1}{2}}}{1-h(\kappa, H)^{\frac{1}{2}}} \mathcal{N}^{n}
$$

where $h$ is introduced in (7.8).

Proof. Recall that $\mathbf{A}_{0}$ is the magnetic potential introduced in (2.2), $\phi_{x_{0}}$ is defined in (5.1) and that, for $\widetilde{x}_{0} \in \overline{Q_{\ell}\left(x_{0}\right)}, \varphi_{x_{0}, \widetilde{x}_{0}}$ is the function satisfying $(3.2)$.

Having in mind the definition of $b$ and $R$ in (7.4) and their properties, and using (2.15), we get from $(5.8)$ and $(5.9)$ the following inequality

$$
\begin{aligned}
\mathcal{E}_{0}\left(\psi, \sigma_{\ell}\left|B_{0}\left(\widetilde{x}_{0}\right)\right| \mathbf{A}_{0}\left(x-x_{0}\right)+\nabla \varphi, Q_{\delta(\kappa)}^{j}\right) & \geq \frac{e_{N}(b, R)}{b} \\
& \geq \frac{R^{2}}{b} \hat{f}(b)(1-\operatorname{err}(b, R)),
\end{aligned}
$$

where $\varphi=\phi_{x_{0}}+\varphi_{x_{0}, \widetilde{x}_{0}}, e_{N}$ is introduced in $(2.3), b=b\left(\kappa, H, \widetilde{x}_{0}\right)$ and $R=R\left(\kappa, H, \widetilde{x}_{0}\right)$.

As a consequence of (7.8), 7.13) becomes

$$
\mathcal{E}_{0}\left(\psi, \sigma_{\ell}\left|B_{0}\left(\widetilde{x}_{0}\right)\right| \mathbf{A}_{0}\left(x-x_{0}\right)+\nabla \varphi, Q_{\delta(\kappa)}^{j}\right) \geq \kappa^{2} \delta(\kappa)^{2} \hat{f}\left(\frac{H}{\kappa}\left|B_{0}\left(\widetilde{x}_{0}\right)\right|\right)(1-h(\kappa, H)) .
$$

Notice that

$$
\left|Q_{\ell}\left(x_{0}\right)\right|=\sum_{j \in \mathcal{J}}\left|Q_{\delta(\kappa)}^{j}\right|=\left(\mathcal{N}^{n}+\mathcal{N}^{b}\right) \delta(\kappa)^{2}
$$

Thus, we may write

$$
\sum_{j \in \mathcal{J}} \mathcal{E}_{0}\left(\psi, \sigma_{\ell}\left|B_{0}\left(\widetilde{x}_{0}\right)\right| \mathbf{A}_{0}\left(x-x_{0}\right)+\nabla \varphi, Q_{\delta(\kappa)}^{j}\right)=\mathcal{E}_{0}\left(\psi, \sigma_{\ell}\left|B_{0}\left(\widetilde{x}_{0}\right)\right| \mathbf{A}_{0}\left(x-x_{0}\right)+\nabla \varphi, Q_{\ell}\left(x_{0}\right)\right) .
$$


When (1.7) is satisfied, we have uniformly

$$
\ell^{2} \kappa H \rho=(\kappa H)^{\frac{1}{2}}\left(\ln \frac{\kappa}{H}\right)^{-\frac{1}{2}} \gg 1, \quad \text { as } \kappa \longrightarrow+\infty .
$$

Hence the assumptions of Proposition 6.3 are satisfied. Putting (7.16) into (6.2), using (7.1) and 7.15, we get

$$
\begin{aligned}
\mid \sum_{j \in \mathcal{J}}\left[\mathcal{E}_{0}\left(\psi, \sigma_{\ell}\left|B_{0}\left(\widetilde{x}_{0}\right)\right| \mathbf{A}_{0}\left(x-x_{0}\right)+\nabla \varphi, Q_{\delta(\kappa)}^{j}\right)\right. & \left.-\kappa^{2} \delta(\kappa)^{2} \hat{f}\left(\frac{H}{\kappa}\left|B_{0}\left(\widetilde{x}_{0}\right)\right|\right)\right] \mid \\
& \leq C\left(\mathcal{N}^{b}+\mathcal{N}^{n}\right) \delta(\kappa)^{2} \kappa H\left(\ln \frac{\kappa}{H}\right)^{\frac{1}{8}} .
\end{aligned}
$$

Using the monotonicity of $\hat{f}$ and remembering that $\left(\ell, x_{0}, \widetilde{x}_{0}\right)$ is a $\rho$-admissible triple, we get,

$$
\hat{f}\left(\frac{H}{\kappa}\left|B_{0}\left(\widetilde{x}_{0}\right)\right|\right) \geq \hat{f}\left(\frac{H}{\kappa} \rho\right) .
$$

Using (2.11), 7.1 and (7.5), we obtain as $\kappa \longrightarrow+\infty$

$$
\begin{aligned}
\kappa^{2} \hat{f}\left(\frac{H}{\kappa} \rho\right) & \geq \kappa H \rho\left(\ln \frac{\kappa}{H \rho}\right)\left(1+\hat{s}\left(\frac{H}{\kappa} \rho\right)\right) \\
& \geq \kappa H \rho\left(\ln \frac{\kappa}{H}+\ln \frac{1}{\rho}\right)\left(1+\hat{s}\left(\frac{H}{\kappa} \rho\right)\right) \\
& \geq \kappa H\left(\ln \frac{\kappa}{H}\right)^{\frac{1}{2}}\left(1+\hat{s}_{1}(\kappa, H)\right)
\end{aligned}
$$

where $\hat{s}_{1}(\kappa, H)=\hat{s}\left(\frac{H}{\kappa}\left(\ln \frac{\kappa}{H}\right)^{-\frac{1}{2}}\right)$ is uniformly $o(1)$ for $H$ satisfying 1.7 ).

Collecting (7.18)-(7.19), we get for $\kappa$ sufficiently large

$$
\begin{aligned}
\kappa^{2} \hat{f}\left(\frac{H}{\kappa}\left|B_{0}\left(\widetilde{x}_{0}\right)\right|\right) & \geq \kappa H\left(\ln \frac{\kappa}{H}\right)^{\frac{1}{2}}\left(1+\hat{s}_{1}(\kappa, H)\right) \\
& \geq \frac{\kappa H}{2}\left(\ln \frac{\kappa}{H}\right)^{\frac{1}{2}} .
\end{aligned}
$$

Multiplying both sides by $\left(\ln \frac{\kappa}{H}\right)^{-\frac{3}{8}}$ and using the definition of $h$ in 7.8 , we get

$$
\kappa^{2} \hat{f}\left(\frac{H}{\kappa}\left|B_{0}\left(\widetilde{x}_{0}\right)\right|\right) h(\kappa, H) \geq \frac{\kappa H}{2}\left(\ln \frac{\kappa}{H}\right)^{\frac{1}{8}} .
$$

Puting (7.22) into 7.17), we obtain

$$
\begin{aligned}
\mid \sum_{j \in \mathcal{J}}\left[\mathcal { E } _ { 0 } \left(\psi, \sigma_{\ell}\left|B_{0}\left(\widetilde{x}_{0}\right)\right| \mathbf{A}_{0}\left(x-x_{0}\right)\right.\right. & \left.\left.+\nabla \varphi, Q_{\delta(\kappa)}^{j}\right)-\kappa^{2} \delta(\kappa)^{2} \hat{f}\left(\frac{H}{\kappa}\left|B_{0}\left(\widetilde{x}_{0}\right)\right|\right)\right] \mid \\
& \leq C\left(\mathcal{N}^{b}+\mathcal{N}^{n}\right) \kappa^{2} \delta(\kappa)^{2} \hat{f}\left(\frac{H}{\kappa}\left|B_{0}\left(\widetilde{x}_{0}\right)\right|\right) h(\kappa, H) .
\end{aligned}
$$


Using (7.11), (7.14) and (7.23), we may write

$$
\begin{aligned}
\mathcal{N}^{b} \kappa^{2} \delta(\kappa)^{2} \hat{f}\left(\frac{H}{\kappa}\left|B_{0}\left(\widetilde{x}_{0}\right)\right|\right) h(\kappa, H)^{\frac{1}{2}} & \\
\leq & \sum_{j \in \mathcal{J}^{b}}\left[\mathcal{E}_{0}\left(\psi, \sigma_{\ell}\left|B_{0}\left(\widetilde{x}_{0}\right)\right| \mathbf{A}_{0}\left(x-x_{0}\right)+\nabla \varphi, Q_{\delta(\kappa)}^{j}\right)-\kappa^{2} \delta(\kappa)^{2} \hat{f}\left(\frac{H}{\kappa}\left|B_{0}\left(\widetilde{x}_{0}\right)\right|\right)\right] \\
\leq & \quad+\sum_{j \in \mathcal{J}}\left[\mathcal { E } _ { 0 } \left(\psi, \sigma_{\ell}\left|B_{0}\left(\widetilde{x}_{0}\right)\right| \mathbf{A}_{0}\left(x-x_{0}\right)+\nabla \varphi, Q_{\delta(\kappa)}^{j} \hat{f}\left(\frac{H}{\kappa}\left|B_{0}\left(\widetilde{x}_{0}\right)\right|\right) h(\kappa, H)\right.\right. \\
& \leq C\left(\mathcal{J}^{2} \delta(\kappa)^{2} \hat{f}\left(\frac{H}{\kappa}\left|B_{0}\left(\widetilde{x}_{0}\right)\right|\right)\right] \\
\leq & h(\kappa, H)+\mathcal{N}^{n} \kappa^{2} \delta(\kappa)^{2} \hat{f}\left(\frac{H}{\kappa}\left|B_{0}\left(\widetilde{x}_{0}\right)\right|\right) h(\kappa, H) .
\end{aligned}
$$

We divide both sides by $\kappa^{2} \delta(\kappa)^{2} \hat{f}\left(\frac{H}{\kappa}\left|B_{0}\left(\widetilde{x}_{0}\right)\right|\right) h(\kappa, H)^{\frac{1}{2}}$ to get 7.12$)$.

Remark 7.2. Using (7.9) and (1.7), we obtain uniformly

$$
\mathcal{N}^{b} \ll \mathcal{N}^{n}, \quad \text { as } \kappa \longrightarrow+\infty .
$$

More precisely, we mean that $\mathcal{N}^{b}=\mathcal{N}^{n} e\left(\kappa, H, \ell, \widetilde{x}_{0}, x_{0}\right)$, with $e\left(\kappa, H, \ell, \widetilde{x}_{0}, x_{0}\right)$ is uniformly $o(1)$ for any $\kappa \geq \kappa_{0}$, any $\rho$-admissible triple $\left(\ell, \widetilde{x}_{0}, x_{0}\right)$, any $H$ satisfying (1.7).

7.3. The results of Sandier-Serfaty. Now we recall an important result of Sandier-Serfaty [11. Define the energy of $(u, A) \in H^{1}(\mathcal{D} ; \mathbb{C}) \times H_{\text {div }}^{1}(\mathcal{D} ; \mathbb{R})$ in a domain $\mathcal{D} \subset \mathbb{R}^{2}$ as follows

$$
J_{\mathcal{D}}(u, A)=\int_{\mathcal{D}}|(\nabla-i A) u|^{2}+\frac{\kappa^{2}}{2}\left(1-|u|^{2}\right)^{2}+\left|\operatorname{curl} A-h_{\mathrm{ex}}\right|^{2} d x .
$$

The next proposition is essentially proved ${ }^{3}$ in [11, Proposition 5.1].

Proposition 7.3. Let $\hat{h}:(0,+\infty) \longrightarrow(0,+\infty)$ such that $\lim _{t \longrightarrow+\infty} \hat{h}(t)=0$, there exist two functions $s_{1}, s_{2}:(0,+\infty) \longrightarrow(0,+\infty)$ satisfying

$$
\lim _{t \longrightarrow+\infty} s_{1}(t)=0, \quad \lim _{t \longrightarrow+\infty} s_{2}(t)=0 .
$$

Assume that $h_{\mathrm{ex}}$ is a function of $\kappa$ and $K$ is a square of side length $\gamma(\kappa)$ such that

$|\ln \kappa| \ll h_{\mathrm{ex}} \ll \kappa^{2} \quad$ and $\quad \ln \frac{\kappa}{\sqrt{h_{\mathrm{ex}}}} \ll h_{\mathrm{ex}} \gamma(\kappa)^{2} \ll \min \left(h_{\mathrm{ex}},\left(\ln \frac{\kappa}{\sqrt{h_{\mathrm{ex}}}}\right)^{2}\right), \quad$ as $\kappa \longrightarrow \infty$.

If $(u, A) \in C^{1}(\bar{K} ; \mathbb{C}) \times C^{1}\left(\bar{K} ; \mathbb{R}^{2}\right)$ verifies

$$
J_{K}(u, A) \leq h_{\mathrm{ex}} \gamma(\kappa)^{2} \ln \frac{\kappa}{\sqrt{h_{\mathrm{ex}}}}(1+\hat{h}(\kappa)),
$$

then, there exist disjoint disks $\left(D\left(a_{i}, r_{i}\right)\right)_{i=1}^{m}$ such that:

(1) $\sum r_{i} \leq h_{\mathrm{ex}}^{-\frac{1}{2}}$

(2) $|u|>\frac{1}{2}$ on $\cup_{i} \partial D\left(a_{i}, r_{i}\right)$

(3) If $d_{i}=\operatorname{deg}\left(\frac{u}{|u|}, \partial D\left(a_{i}, r_{i}\right)\right)$, then, as $\kappa \longrightarrow+\infty$

\footnotetext{
${ }^{3}$ We replaced $\varepsilon$ by $\frac{1}{\kappa}$. We can indeed verify that only the upper bound of $J_{K}(u, A)$ is needed with no additional condition on $f(\varepsilon)$ and that the $o(1)$ are actually uniform under uniform assumptions. Note also that we do not use in this proposition that $(u, A)$ is a critical point of $J_{\Omega}$.
} 


$$
2 \pi \sum_{i=1}^{m} d_{i} \geq h_{\mathrm{ex}} \gamma(\kappa)^{2}\left(1-s_{1}(\kappa)\right) \quad \text { and } \quad 2 \pi \sum_{i=1}^{m}\left|d_{i}\right| \leq h_{\mathrm{ex}} \gamma(\kappa)^{2}\left(1+s_{2}(\kappa)\right) .
$$

The next lemma will give us that $\delta(\kappa)$, the side length of the square $Q_{\delta(\kappa)}^{j}$, satisfies (7.27) and will be useful in Proposition 7.5 .

Lemma 7.4. Under the assumptions of the previous subsection we have

$$
\begin{aligned}
& \delta(\kappa)^{2}=\frac{1}{\varepsilon_{1}\left(\kappa, \ell, x_{0}, \widetilde{x}_{0}, H\right)} \frac{1}{\kappa H\left|B_{0}\left(\widetilde{x}_{0}\right)\right|} \ln \frac{\kappa}{H\left|B_{0}\left(\widetilde{x}_{0}\right)\right|}, \quad \text { as } \kappa \longrightarrow+\infty \\
& \delta(\kappa)^{2}=\varepsilon_{2}\left(\kappa, \ell, x_{0}, \widetilde{x}_{0}, H\right) \frac{1}{\kappa H\left|B_{0}\left(\widetilde{x}_{0}\right)\right|}\left(\ln \frac{\kappa}{H\left|B_{0}\left(\widetilde{x}_{0}\right)\right|}\right)^{2}, \quad \text { as } \kappa \longrightarrow+\infty,
\end{aligned}
$$

where $\varepsilon_{1}\left(\kappa, \ell, x_{0}, \widetilde{x}_{0}, H\right)$ and $\varepsilon_{2}\left(\kappa, \ell, x_{0}, \widetilde{x}_{0}, H\right)$ are uniformly o(1) as $\kappa \longrightarrow+\infty$.

Proof.

Proof of 7.30. We know that for all $\rho$-admissible triple $\left(\ell, x_{0}, \widetilde{x}_{0}\right)$

$$
\frac{1}{\kappa H\left|B_{0}\left(\widetilde{x}_{0}\right)\right|} \ln \frac{\kappa}{H\left|B_{0}\left(\widetilde{x}_{0}\right)\right|} \leq \frac{1}{\kappa H \rho} \ln \frac{\kappa}{H \rho} \leq \frac{3}{2} \frac{1}{\kappa H}\left(\ln \frac{\kappa}{H}\right)^{\frac{3}{2}},
$$

Also, we know that

$$
\frac{1}{\kappa H}\left(\ln \frac{\kappa}{H}\right)^{\frac{3}{2}} \ll 2^{-\frac{7}{4}} \frac{1}{\kappa H}\left(\ln \frac{\kappa}{H}\right)^{\frac{7}{4}} \sim \delta(\kappa)^{2} .
$$

Proof of 7.31. On the other hand, we have

$$
\frac{1}{\kappa H\left|B_{0}\left(\widetilde{x}_{0}\right)\right|}\left(\ln \frac{\kappa}{H\left|B_{0}\left(\widetilde{x}_{0}\right)\right|}\right)^{2} \geq \frac{1}{\kappa H \bar{\beta}_{0}}\left(\ln \frac{\kappa}{H \bar{\beta}_{0}}\right)^{2} \geq C \frac{1}{\kappa H}\left(\ln \frac{\kappa}{H}\right)^{2},
$$

where $C$ is a positive constant and $\bar{\beta}_{0}$ is introduced in 7.6 .

It is clear that

$$
\frac{1}{\kappa H}\left(\ln \frac{\kappa}{H}\right)^{2} \gg 2^{-\frac{7}{4}} \frac{1}{\kappa H}\left(\ln \frac{\kappa}{H}\right)^{\frac{7}{4}} \sim \delta(\kappa)^{2} .
$$

We can prove the following result regarding the vortices of the minimizers in the 'nice squares'. We start with the admissible squares contained in $\Omega \cap\left\{B_{0}>0\right\}$.

Proposition 7.5. Under Assumptions (1.2) and (1.7) there exists $s_{1}, s_{2}:(0,+\infty) \longrightarrow(0,+\infty)$ two functions satisfying (7.26) and such that, for any $\left(\ell, x_{0}, \widetilde{x}_{0}\right)$ such that $\overline{Q_{\delta(\kappa)}^{j}} \subset \Omega \cap\left\{B_{0}>\rho\right\}$ and $\widetilde{x}_{0} \in \overline{Q_{\delta(\kappa)}^{j}}$ for which $Q_{\delta(\kappa)}^{j}$ is a nice square, and any minimizer $(\psi, \mathbf{A}) \in H^{1}(\Omega, \mathbb{C}) \times$ $H_{\text {div }}^{1}\left(\Omega, \mathbb{R}^{2}\right)$ of 1.1 , there exist disjoint disks $\left(D\left(a_{i, j}, r_{i, j}\right)\right)_{i=1}^{m_{j}}$ in $Q_{\delta(\kappa)}^{j}$ such that

- $\sum_{i=1}^{m_{j}} r_{i, j} \leq\left(\kappa H B_{0}\left(\widetilde{x}_{0}\right)\right)^{-\frac{1}{2}}$

- $|\psi|>\frac{1}{2}$ on $\cup_{j} \partial D\left(a_{i, j}, r_{i, j}\right)$

- If $d_{i, j}$ is the winding number of $\frac{\psi}{|\psi|}$ restricted to $\partial D\left(a_{i, j}, r_{i, j}\right)$, then

$$
2 \pi \sum_{i=1}^{m_{j}} d_{i, j} \geq \delta(\kappa)^{2} \kappa H B_{0}\left(\widetilde{x}_{0}\right)\left(1-s_{1}(\kappa)\right), \quad \text { as } \kappa \longrightarrow+\infty,
$$

and

$$
2 \pi \sum_{i=1}^{m_{j}}\left|d_{i, j}\right| \leq \delta(\kappa)^{2} \kappa H B_{0}\left(\widetilde{x}_{0}\right)\left(1+s_{2}(\kappa)\right), \quad \text { as } \kappa \longrightarrow+\infty \text {. }
$$


Proof.

We will apply Proposition 7.3 with

$$
K=Q_{\delta(\kappa)}^{j}, \gamma(\kappa)=\delta(\kappa), h_{\mathrm{ex}}=\kappa H B_{0}\left(\widetilde{x}_{0}\right), u=e^{-i \kappa H \varphi} \psi \text { and } A(x)=\kappa H B_{0}\left(\widetilde{x}_{0}\right) \mathbf{A}_{0}\left(x-x_{0}\right),
$$

where $\mathbf{A}_{0}$ is the magnetic potential introduced in $(2.2)$ and $\varphi=\phi_{x_{0}}+\varphi_{x_{0}, \widetilde{x}_{0}}$, with $\phi_{x_{0}}$ defined in (5.1) and $\varphi_{x_{0}, \widetilde{x}_{0}}$ in (3.2).

Let us verify that the conditions of the proposition are satisfied for this choice.

First, we start by proving 7.28 . Since curl $\mathbf{A}_{0}=1$, then,

$$
\operatorname{curl} A=\kappa H B_{0}\left(\widetilde{x}_{0}\right)=h_{\mathrm{ex}},
$$

and consequently

$$
\int_{Q_{\delta(\kappa)}^{j}}\left|\operatorname{curl} A-h_{\mathrm{ex}}\right|^{2} d x=0
$$

This implies that, for any $j \in \mathcal{J}^{n}$

$$
\begin{aligned}
J_{K}(u, A) & =\int_{Q_{\delta(\kappa)}^{j}}\left(\left|\left(\nabla-i \kappa H\left(B_{0}\left(\widetilde{x}_{0}\right) \mathbf{A}_{0}\left(x-x_{0}\right)+\nabla \varphi\right)\right) \psi\right|^{2}+\frac{\kappa^{2}}{2}\left(1-|\psi|^{2}\right)^{2}\right) d x \\
& =\mathcal{E}_{0}\left(\psi, B_{0}\left(\widetilde{x}_{0}\right) \mathbf{A}_{0}\left(x-x_{0}\right)+\nabla \varphi, Q_{\delta(\kappa)}^{j}\right) .
\end{aligned}
$$

Since $Q_{\delta(\kappa)}^{j}$ is a nice square, then,

$$
J_{K}(u, A) \leq \delta(\kappa)^{2} \kappa^{2} \hat{f}\left(\frac{H}{\kappa} B_{0}\left(\widetilde{x}_{0}\right)\right)\left(1+h(\kappa, H)^{\frac{1}{2}}\right), \quad \text { as } \kappa \longrightarrow+\infty .
$$

As a consequence of (2.11), (7.38) becomes

$$
J_{K}(u, A) \leq \frac{1}{2} \delta(\kappa)^{2} \kappa H B_{0}\left(\widetilde{x}_{0}\right) \ln \frac{\kappa}{H B_{0}\left(\widetilde{x}_{0}\right)}(1+\hat{h}(\kappa, H)), \quad \text { as } \kappa \longrightarrow+\infty,
$$

where

$$
\hat{h}(\kappa, H)=h(\kappa, H)^{\frac{1}{2}}+\hat{s}\left(\frac{H}{\kappa}\right)+\hat{s}\left(\frac{H}{\kappa}\right) h(\kappa, H)^{\frac{1}{2}} .
$$

Notice that the function $\hat{h}(\kappa, H)$ is uniformly $o(1)$ as $\kappa \longrightarrow+\infty$ and $H$ satisfying (1.7).

Secondly we prove (7.27). In fact, under Assumption (1.7), we can easly prove, uniformly as $\kappa \longrightarrow+\infty$,

$$
|\ln \kappa| \ll h_{\mathrm{ex}} \ll \kappa^{2},
$$

and

$$
\min \left(h_{\mathrm{ex}},\left(\ln \frac{\kappa}{\sqrt{h_{\mathrm{ex}}}}\right)^{2}\right)=\left(\ln \frac{\kappa}{\sqrt{h_{\mathrm{ex}}}}\right)^{2} .
$$

Thanks to Lemma 7.4, we get that 7.27) is satisfied and in this way we achieve the proof of Proposition 7.5 .

In light of Lemma 7.1, we deduce from Proposition 7.5 the distribution of vortices in a $\rho$ admissible square $Q_{\ell}$.

Proposition 7.6. Suppose that Assumptions (1.2) and 1.7 are true. There exists two functions $s_{1}, s_{2}:(0 ;+\infty) \longrightarrow(0 ;+\infty)$ satisfying 7.26$)$ and the following is true. Let $(\psi, \mathbf{A}) \in H^{1}(\Omega, \mathbb{C}) \times$ 
$H_{\text {div }}^{1}\left(\Omega, \mathbb{R}^{2}\right)$ be a minimizer of 11.1 and $\left(\ell, x_{0}\right)$ such that $\overline{Q_{\ell}\left(x_{0}\right)} \subset \Omega \cap\left\{B_{0}>\rho\right\}$. There exist a family of disjoint disks (indexed by $\left.\mathcal{K}=\mathcal{K}_{\ell, x_{0}}\right)\left(D\left(\widetilde{a}_{k}, \widetilde{r}_{k}\right)\right)_{k \in \mathcal{K}}$ in $Q_{\ell}\left(x_{0}\right)$ such that

- $\sum_{k \in \mathcal{K}} \widetilde{r}_{k} \leq\left(\kappa H B_{0}\left(\widetilde{x}_{0}\right)\right)^{-\frac{1}{2}}\left(\frac{\ell}{\delta(\kappa)}\right)^{2}(1+o(1)), \quad$ as $\kappa \longrightarrow+\infty$

- $|\psi|>\frac{1}{2}$ on $\cup_{k} \partial D\left(\widetilde{a}_{k}, \widetilde{r}_{k}\right)$

- If $\widetilde{d}_{k}$ is the winding number of $\frac{\psi}{|\psi|}$ restricted to $\partial D\left(\widetilde{a}_{k}, \widetilde{r}_{k}\right)$, then as $\kappa \longrightarrow+\infty$

$$
2 \pi \sum_{k \in \mathcal{K}} \widetilde{d}_{k} \geq \ell^{2} \kappa H B_{0}\left(\widetilde{x}_{0}\right)\left(1-s_{1}(\kappa)\right) \quad \text { and } \quad 2 \pi \sum_{k \in \mathcal{K}}\left|\widetilde{d}_{k}\right| \leq \ell^{2} \kappa H B_{0}\left(\widetilde{x}_{0}\right)\left(1+s_{2}(\kappa)\right) .
$$

Here, the function o(1) is bounded independently of the choice of $\widetilde{x}_{0}$ and the minimizer $(\psi, \mathbf{A})$.

Proof. Recall that $Q_{\ell}\left(x_{0}\right)$ is decomposed into $\mathcal{N}^{n}$ 'nice squares' $\left(Q_{\delta(k)}^{j}\right)_{j \in \mathcal{J}^{n}}$ and $\mathcal{N}^{b}$ 'bad squares' $\left(Q_{\delta(k)}^{j}\right)_{j \in \mathcal{J}^{b}}$.

In every nice square $Q_{\delta(\kappa)}^{j}$, Proposition 7.5 tells us that there exist disjoint disks $\left(D\left(a_{i, j}, r_{i, j}\right)\right)_{i=1}^{m_{j}}$ such that (7.32), (7.33) and (7.34) hold. Let $\left(D\left(\widetilde{a}_{k}, \widetilde{r}_{k}\right)\right)_{k \in \mathcal{K}}=\left(D\left(a_{i, j}, r_{i, j}\right)\right)_{i, j}$ be the family of disjoint disks in $\cup_{j \in \mathcal{J}^{n}} Q_{\delta(\kappa)}^{j}$. Clearly

$$
\sum_{k \in \mathcal{K}} \widetilde{r}_{k}=\sum_{j \in \mathcal{J}^{n}} \sum_{i=1}^{m_{j}} r_{i, j} .
$$

This implies that

$$
\sum_{k \in \mathcal{K}} \widetilde{r}_{k} \leq\left(\kappa H B_{0}\left(\widetilde{x}_{0}\right)\right)^{-\frac{1}{2}} \mathcal{N}^{n}
$$

Having in mind 7.15 and 7.24 , we have, as $\kappa \longrightarrow+\infty$,

$$
\mathcal{N}^{n} \frac{\delta(\kappa)^{2}}{\ell^{2}} \longrightarrow 1
$$

This implies that

$$
\sum_{k \in \mathcal{K}} \widetilde{r}_{k} \leq\left(\kappa H B_{0}\left(\widetilde{x}_{0}\right)\right)^{-\frac{1}{2}}\left(\frac{\ell}{\delta(\kappa)}\right)^{2}(1+o(1)),
$$

where the function $o(1)$ is bounded independently of the choice of $\widetilde{x}_{0}$.

Let $\widetilde{d}_{k}$ be the winding number of $\frac{\psi}{|\psi|}$ restricted to $\partial D\left(\widetilde{a}_{k}, \widetilde{r}_{k}\right)$, then, for any $k \in \mathcal{K}$

$$
\sum_{k \in \mathcal{K}} \widetilde{d}_{k}=\sum_{j \in \mathcal{J}^{n}} \sum_{i=1}^{m_{j}} d_{i, j} .
$$

Since from (7.33, (7.34) and 7.43, we get, as $\kappa \longrightarrow+\infty$,

$$
\begin{aligned}
2 \pi \sum_{k \in \mathcal{K}} \widetilde{d}_{k}=2 \pi \sum_{j \in \mathcal{J}^{n}} \sum_{i=1}^{m_{j}} d_{i, j} & \geq \mathcal{N}^{n} \delta(\kappa)^{2} \kappa H B_{0}\left(\widetilde{x}_{0}\right)\left(1-s_{1}(\kappa)\right) \\
& \geq \ell^{2} \kappa H B_{0}\left(\widetilde{x}_{0}\right)\left(1-s_{1}(\kappa)\right)
\end{aligned}
$$


and

$$
\begin{aligned}
2 \pi \sum_{k \in \mathcal{K}}\left|\widetilde{d}_{k}\right|=2 \pi \sum_{j \in \mathcal{J}^{n}} \sum_{i=1}^{m_{j}}\left|d_{i, j}\right| & \leq \mathcal{N}^{n} \delta(\kappa)^{2} \kappa H B_{0}\left(\widetilde{x}_{0}\right)\left(1+s_{2}(\kappa)\right) \\
& \leq \ell^{2} \kappa H B_{0}\left(\widetilde{x}_{0}\right)\left(1+s_{2}(\kappa)\right) .
\end{aligned}
$$

This finishes the proof of Proposition 7.6.

7.4. Proof of Theorem 1.6. Let $(\psi, \mathbf{A}) \in H^{1}(\Omega, \mathbb{C}) \times H_{\text {div }}^{1}\left(\Omega, \mathbb{R}^{2}\right)$ be a minimizer of (1.1) and $\Gamma_{\ell}:=\ell \mathbb{Z} \times \ell \mathbb{Z}$ a lattice of $\mathbb{R}^{2}$. For all $\gamma \in \Gamma_{\ell}$, we consider the family of squares $Q_{\ell}(\gamma)$ and $\widetilde{\gamma} \in Q_{\ell}(\gamma)$. Consider an open set $S \subset \Omega \cap\left\{B_{0}>0\right\}$ such that the boundary of $S$ is smooth. Let

$$
\begin{gathered}
\mathcal{J}_{\ell}=\left\{\gamma ; \quad \overline{Q_{\ell}(\gamma)} \subset S \cap\left\{B_{0}>\rho\right\}\right\}, \\
\mathcal{M}=\operatorname{card} \mathcal{J}_{\ell},
\end{gathered}
$$

and

$$
S_{\ell}=\operatorname{int}\left(\cup_{\gamma \in \mathcal{J}_{\ell}} \overline{Q_{\ell}(\gamma)}\right)
$$

Then, as $\kappa \longrightarrow+\infty$, we have

$$
\mathcal{M} \times \ell^{2} \longrightarrow|S|
$$

Proof of (3):

Proposition 7.6 tells us that there exist disjoint disks $\left(D\left(\widetilde{a}_{k, \gamma}, \widetilde{r}_{k, \gamma}\right)\right)_{k \in \mathcal{K}_{\ell, \gamma}}$ in each square $Q_{\ell}(\gamma)$ with $\overline{Q_{\ell}(\gamma)} \subset \Omega \cap\left\{B_{0}>\rho\right\}$ such that 7.40 and 7.42 hold. We introduce the measure by

$$
\mu_{\kappa}:=\frac{2 \pi}{\kappa H} \sum_{\gamma \in \mathcal{J}_{\ell}} \sum_{k \in \mathcal{K}_{\ell, \gamma}} \widetilde{d}_{k, \gamma} \delta_{\widetilde{a}_{k, \gamma}},
$$

where $\widetilde{d}_{k, \gamma}$ is the winding number introduced before 7.42 and $\delta_{\widetilde{a}_{k, \gamma}}$ is the unit Dirac mass at $\widetilde{a}_{k, \gamma}$.

Having in mind 7.42 we have for any $(\ell, \gamma, \widetilde{\gamma})$ such that $\overline{Q_{\ell}(\gamma)} \subset \Omega \cap\left\{B_{0}>\rho\right\}$ and $\widetilde{\gamma} \in \overline{Q_{\ell}(\gamma)}$

$$
B_{0}(\widetilde{\gamma}) \ell^{2}\left(1-s_{1}(\kappa)\right) \leq \frac{2 \pi}{\kappa H} \sum_{k \in \mathcal{K}_{\ell, \gamma}} \widetilde{d}_{k, \gamma} \leq B_{0}(\widetilde{\gamma}) \ell^{2}\left(1+s_{2}(\kappa)\right)
$$

Using (7.49), we obtain

$$
\left(\sum_{\gamma \in \mathcal{J}_{\ell}} B_{0}(\widetilde{\gamma}) \ell^{2}\right)-2 \bar{\beta}_{0}|S| s_{1}(\kappa) \leq \frac{2 \pi}{\kappa H} \sum_{\gamma \in \mathcal{J}_{\ell}} \sum_{k \in \mathcal{K}_{\ell, \gamma}} \widetilde{d}_{k, \gamma} \leq\left(\sum_{\gamma \in \mathcal{J}_{\ell}} B_{0}(\widetilde{\gamma}) \ell^{2}\right)+2 \bar{\beta}_{0}|S| s_{2}(\kappa) .
$$

Here, we have used the fact that $B_{0}(\widetilde{\gamma}) \leq \bar{\beta}_{0}$ to estimate the errors terms, where $\bar{\beta}_{0}$ is introduced in (7.6).

Now it is time to determine $\sum_{\gamma \in \mathcal{J}_{\ell}} B_{0}(\widetilde{\gamma}) \ell^{2}$. We will do this in two steps:

Upper bound: Notice that till now $\widetilde{\gamma}$ was an arbitrary point in $Q_{\ell}(\gamma)$, but that our estimates are independent of this choice. We now select $\widetilde{\gamma} \in \overline{Q_{\ell}(\gamma)}$ such that $B_{0}(\widetilde{\gamma})=\underline{B}_{\gamma, \ell}$ with $\underline{B}_{\gamma, \ell}$ satisfying (3.13), and get

$$
\sum_{\gamma \in \mathcal{J}_{\ell}} B_{0}(\widetilde{\gamma}) \ell^{2}=\sum_{\gamma \in \mathcal{J}_{\ell}} \underline{B}_{\gamma, \ell^{\ell}}{ }^{2}
$$

We recognize in the right hand side above the lower Riemann sum of $x \longrightarrow B_{0}(x)$ and we use that $S_{\ell} \subset S$ to obtain

$$
\sum_{\gamma \in \mathcal{J}_{\ell}} B_{0}(\widetilde{\gamma}) \ell^{2} \leq \int_{S} B_{0}(x) d x
$$


Lower bound: We select $\widetilde{\gamma} \in Q_{\ell}(\gamma)$ such that $B_{0}(\widetilde{\gamma})=\bar{B}_{\gamma, \ell}$ with $\bar{B}_{\gamma, \ell}$ satisfies (5.17). Similarly to what we did in the upper bound above, we get

$$
\sum_{\gamma \in \mathcal{J}_{\ell}} B_{0}(\widetilde{\gamma}) \ell^{2} \geq \int_{S_{\ell}} B_{0}(x) d x
$$

Notice that using the regularity of $\partial S$ and 1.2 , we have as $\kappa \longrightarrow+\infty$

$$
\left|S \backslash S_{\ell}\right|=\mathcal{O}(\ell|\partial S|) .
$$

Therefore

$$
\begin{aligned}
\int_{S_{\ell}} B_{0}(x) d x & =\int_{S} B_{0}(x) d x-\int_{S \backslash S_{\ell}} B_{0}(x) d x \\
& \geq \int_{S} B_{0}(x) d x-\bar{\beta}_{0}\left|S \backslash S_{\ell}\right| \\
& \geq \int_{S} B_{0}(x) d x-C \ell,
\end{aligned}
$$

where $\bar{\beta}_{0}$ is introduced in 7.6 and $C$ is a positive constant.

This implies that

$$
\sum_{\gamma \in \mathcal{J}_{\ell}} B_{0}(\widetilde{\gamma}) \ell^{2} \geq \int_{S} B_{0}(x) d x-C \ell .
$$

The estimates in 7.52 and 7.54 allow us to deduce from (7.51) that

$$
-C \ell-2 \bar{\beta}_{0}|S| s_{1}(\kappa) \leq \mu_{\kappa}(S)-\int_{S} B_{0}(x) d x \leq+2 \bar{\beta}_{0}|S| s_{2}(\kappa) .
$$

Consequently, as $\kappa \longrightarrow+\infty$

$$
\mu_{\kappa}(S) \longrightarrow \int_{S} B_{0}(x) d x, \quad \forall S \subset \Omega \cap\left\{B_{0}>0\right\} .
$$

In light of $(7.56)$, we can easily show that $\mu_{\kappa}$ converge weakly to $\mu=B_{0}(x) d x$, which means that:

$$
\mu_{\kappa}(f) \longrightarrow \mu(f), \quad \forall f \in C_{0}\left(\Omega \cap\left\{B_{0}>0\right\}\right) .
$$

Proof of (1): We will prove that the sum of the radii of the disks $\left(D\left(\widetilde{a}_{k, \gamma}, \widetilde{r}_{k, \gamma}\right)\right)_{k \in \mathcal{K}_{\ell, \gamma} \gamma \in \mathcal{J}_{\ell}}$ is less than

$$
(\kappa H)^{\frac{1}{2}}\left(\ln \frac{\kappa}{H}\right)^{-\frac{7}{4}} \int_{S} \frac{1}{\sqrt{B_{0}(x)}} d x(1+o(1)) .
$$

In fact, remembering the choice of $\delta(\kappa)$ in 7.3, , 7.49) and that $\overline{Q_{\ell}(\gamma)} \subset \Omega \cap\left\{B_{0}>0\right\}$, we have

$$
\begin{aligned}
\sum_{\gamma \in \mathcal{J}_{\ell}} \widetilde{r}_{k, \gamma} & =\sum_{\gamma \in \mathcal{J}_{\ell}} \sum_{k \in \mathcal{K}} \widetilde{r}_{k} \\
& \leq(\kappa H)^{\frac{1}{2}}\left(\ln \frac{\kappa}{H}\right)^{-\frac{7}{4}} \sum_{\gamma \in \mathcal{J}_{\ell}} \frac{1}{\sqrt{B_{0}(\widetilde{\gamma})}} \ell^{2}(1+o(1)) .
\end{aligned}
$$

We select $\widetilde{\gamma} \in \overline{Q_{\ell}(\gamma)}$ such that

$$
\frac{1}{\sqrt{B_{0}(\widetilde{\gamma})}}=\inf _{\widehat{\gamma} \in Q_{\gamma, \ell}} \frac{1}{\sqrt{B_{0}(\widehat{\gamma})}},
$$

and we recognize in the right hand side of 7.57 the lower Riemann sum of $x \longrightarrow \frac{1}{\sqrt{B_{0}(x)}}$, we get

$$
\sum_{\gamma \in \mathcal{J}_{\ell}} \widetilde{r}_{k, \gamma} \leq(\kappa H)^{\frac{1}{2}}\left(\ln \frac{\kappa}{H}\right)^{-\frac{7}{4}} \int_{S} \frac{1}{\sqrt{B_{0}(x)}} d x(1+o(1)) .
$$


End of the proof of Theorem 1.6. In $\left\{B_{0}<0\right\} \cap \Omega$, we apply Proposition 7.3 with

$$
K=Q_{\delta(\kappa)}^{j}, \gamma(\kappa)=\delta(\kappa), h_{\mathrm{ex}}=-\kappa H B_{0}\left(\widetilde{x}_{0}\right), u=e^{i \kappa H \varphi} \bar{\psi} \text { and } A(x)=-\kappa H B_{0}\left(\widetilde{x}_{0}\right) \mathbf{A}_{0}\left(x-x_{0}\right) .
$$

So we get that, the convergence of mesure $\mu_{\kappa}$ in 7.56 is still true when $S \subset \Omega \cap\left\{B_{0}<0\right\}$. Similarly, we can control the convergence of $\left|\mu_{\kappa}\right|(S)$. Now we observe that the support of $\mu_{\kappa}$ does not meet $\left\{B_{0}=0\right\}$. Hence $\mu_{\kappa}(S)=\mu_{\kappa}\left(S \cap\left\{B_{0}<0\right\}\right)+\mu_{\kappa}\left(S \cap\left\{B_{0}>0\right\}\right)$ and we can apply the previous arguments to $S_{-}=S \cap\left\{B_{0}<0\right\}$ and $S_{+}=S \cap\left\{B_{0}>0\right\}$.

\section{ACKNOWLEDGEMENTS}

This work is partially supported by a grant from Lebanese University. I would like to thank my supervisors B.Helffer and A.Kachmar for their support, E.Sandier for discussions around vortices and S.Fournais for his help in the proof of Proposition 2.4 .

\section{REFERENCES}

[1] A. Aftalion, S. Serfaty, Lowest Landau level approach in superconductivity for the Abrikosov lattice close to $H_{C_{2}}$, Ann.Inst. H. Poincaré Anal. Non Linéaire, vol.23 (2) 237-267 (2007).

[2] K. Attar, The ground state energy of the two-dimensional Ginzburg-Landau functional with variable magnetic field. To appear in Annales Inst. H. Poincaré Anal. Non Linéaire (2014).

[3] H. Aydi, E. Sandier, Vortex analysis of the periodic Ginzburg-Landau model, Ann. Inst. H. Poincaré Anal. Non Linéaire, vol. 26 (4), 1223-1236 (2009).

[4] S. Fournais, B. Helffer. Optimal uniform elliptic estimates for the Ginzburg-Landau system. Adventures in Mathematical Physics, Contemp. Math. Vol. 447, Amer. Math. Soc. (2007), 83-102.

[5] S. Fournais, B. Helffer, Spectral Methods in Surface Superconductivity. Progr. Nonlinear Differential Equations Appl., vol. 77, Birkhäuser, Boston, (2010).

[6] S. Fournais, A. Kachmar, The ground state energy of the three dimensional Ginzburg-Landau functional Part I: Bulk regime. Comm. Partial Differential Equations. vol. 38 (2), 339-383 (2013).

[7] B. Helffer, A. Kachmar, The Ginzburg-Landau functional with vanishing magnetic field. Preprint.

[8] A. Kachmar, The ground state energy of the three-dimensional Ginzburg-Landau model in the mixed phase. Journal of Functional Analysis. vol. 261, 3328-3344 (2011).

[9] E. Sandier, S. Serfaty, Vortices in the Magnetic Ginzburg-Landau Model. Progr. Nonlinear Differential Equations. Appl., vol. 70, Birkhäuser, Boston, (2007).

[10] E. Sandier, S. Serfaty, The decrease of bulk-superconductivity close to the second critical field in the Ginzburg-Landau model, SIAM J. Math Anal . vol. 34 (4), 939-956 (2010).

[11] E. Sandier, S. Serfaty, On the energy of type-II superconductors in the mixed phase. Rev. in Mathematical Physics. vol. 12 (9), 1219-1257 (2000).

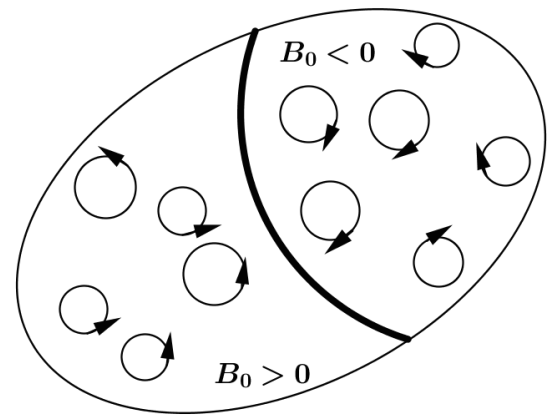

$\Omega$

FiguRE 1. Vortices 